\title{
Emulating the perceptual capabilities of a human evaluator to map the GRB scale for the assessment of voice disorders
}

\author{
J.A. Gómez-García ，L. Moro-Velázquez ，J. Mendes-Laureano ，G. Castellanos-Dominguez , \\ J.I. Godino-Llorente
}

\author{
Keywords: \\ Automatic voice quality analysis \\ GRBAS scale \\ Voice assessment \\ Breathiness \\ Roughness \\ Hoarseness
}

\begin{abstract}
This paper presents the design of an automatic voice quality analysis system for the assessment of voice pathologies, which emulates the perceptual capabilities of a human evaluator according the GRB scale. For this purpose, a novel methodology based on multiple sets of characteristics, ordinal classification and Gaussian regression is proposed. In particular, a reduced subset of characteristics is identified, and the regressor is used to convert the discrete perceptual scale to a continuum, more in agreement to the nature of the problem under study. The robustness of the system is evaluated in several cross-dataset experiments. Similarly, a clinical evaluation of the predictions provided by the system is carried out. Results indicate that the proposed methodology is proficient in modelling the perceptual capabilities of the human evaluator. They also show that it is possible to extend the GRB scale to a continuum through regression techniques while maintaining the consistency of the results. On average, the deviation between the labels assessed by the expert and the ones provided by the system is of about 0.5 units (in a scale from 0 to 3 ) for $G$ and $B$, and of 0.7 units for $R$. Similarly, the deviation of the labels predicted by the system in the clinical assessment trials is about 0.3 units for $G, 0.4$ units for $B$, and 0.5 units for $R$.
\end{abstract}

\section{Introduction}

The clinical evaluation of voice often relies on an instrumental examination and a perceptual analysis. The instrumental medical examination focuses on a primary aetiological diagnosis through the investigation of acoustic, aerodynamic, electroglottographic, videolaryngostroboscopic and/or the exploration of other types of biosignals; whereas the perceptual examination evaluates aspects that are not instrumentally quantifiable, by means of a qualitative description of the perceived degree of dysphonia that is present in the voice (Anniko et al., 2010; Awan et al., 2014). This information might be complemented by a self-assessment where the patient states his/her symptoms (Vaiciukynas et al., 2015), the examination of his/her medical records, evaluation of other body functions and systems, etc. Due to its simplicity, the perceptual evaluation is the most common tool in the clinical setting, being often considered a gold standard to document voice disorders. This perceptual assessment task is generally performed in concordance to a rating scale that evaluates voice quality and provides information about the degree of dysphonia. In particular, one of the most popular perceptual scales is the so called GRBAS scale, which is composed of five categorical descriptors ranging from 0 to 3 , where 0 refers to a healthy condition, 1 to light disease, 2 to moderate impairment and 3 to grave disorder. Each descriptor in the scale measures a distinctive vocal characteristic in such a manner that Anniko et al. (2010): (i) $G$ is a measure of global voice quality or hoarseness; (ii) $R$ is linked to roughness or the level of perceptually audible impressions of irregular glottal pulses, abnormal fluctuations of fundamental frequency or multiple impulses perceived separately (dyplophonia or voice breaks); (iii) $B$ is related to breathiness, or the perceived loss of air due to an incomplete glottal closure and which might include aphonic moments; (iv) $A$ is associated to asthenia, or the weakness or lack of power in voice; and (v) $S$ is linked to strain, or the perceived level of vocal effort. A simplified scale limited to $G, R$ and $B$, named $G R B$, is frequently found in the literature due to the unreliability of the $A$ and $S$ parameters (Moers et al., 2012; Gould et al., 2012). This reduced scale resembles the $R B H$ rating scale used in German-speaking 
countries (Dejonckere, 2009), which encompasses the most important aspects for voice quality assessment.

While the perceptual scales have been designed to evaluate diverse aspects that are regarded relevant for the analysis of voice quality, literature reports large variations in intra- and inter-rater evaluations that compromise the assessments (Kreiman et al., 1993; Barsties and De Bodt, 2015). Indeed, the reliability and accuracy of perceptual evaluations is conditioned mainly by the three factors enumerated next (Barsties and De Bodt, 2015). (i) evaluator factor: includes the number of raters assessing the voice, inherent factors related with the evaluators (e.g. lapses in memory, attention, fatigue, mood state, and mistakes), experience, professional background (e.g. otolaryngologists, speech-language-pathologists, etc.), listener bias due to the potential knowledge of the medical diagnosis, musical background, etc.; (ii) the stimulus: use of anchor stimuli while rating (i.e., reference pattern), the drift in ratings caused by perceptual context, the type of speech task (running speech or sustained vowels), etc.; (iii) the rating scale: equalappearing interval vs. visual analogue scales, ordinal vs. continuous scale, etc.

The increasing need of improving diagnosis and decreasing subjectivity of the perceptual assessment task, has given rise to an emerging field called Automatic Voice Condition Analysis (AVCA) that aims at analysing, classifying and quantifying the degree to which a patient is affected by a voice disorder by means of pattern recognition tools. This analysis is performed using automatic systems that provide objective measurements of the patient's voice, exploiting the close relationship that exists between acoustic features extracted from the speech and voice pathology (Awan et al., 2009). This reduces the evaluation time and the cost of diagnosis and treatment, providing extra advantages such as the avoidance of invasive procedures thanks to the employment of speech signals which are easily recorded by inexpensive means (Godino-Llorente et al., 2006). It has to be remarked though, that the task dealt by these systems is extremely complicated due to the inherent difficulty of the assessment procedure itself and the inherent complexity of the speech signals. Indeed, voice quality has been regarded as a multidimensional construct (Barsties and De Bodt, 2015), which requires several descriptors to characterise its properties. The rating scales on which these systems rely on, have been criticised due to the difficulties in isolating single perceptual dimensions such as breathiness and roughness (Kreiman and Gerratt, 2010). Besides that, the aforementioned inter and intra-rater variability are known factors affecting performance of these systems. Moreover, there are strong differences in the frequency of occurrence of the different labels, which is reflected in the corpora used for training, making the analysis more difficult.

As a consequence, and from a pattern recognition perspective, the AVCA system should consider a multidimensional approach capable of correctly characterising dysphonic voices, following a robust decision making approach, aiming to map these features to the fuzzy labels provided by the evaluators. In addition, the decision making should account for the ordinal nature of the scales and the unbalance training data. Moreover, and since these labels are rated on a four-point scale, they are insensitive to other subtle but potentially informative states inbetween labels (Gould et al., 2012). Thus, the system should consider the existence of more than four states characterising the voices under analysis. Finally, the AVCA system has to be able to manage variability factors that are intrinsic to the clinical setting such as environmental recording conditions, characteristics of the recording equipment, the recording protocols used, individual variability in acoustic and aerodynamic parameters, and the degree of severity of the dysphonia (Oates, 2009).

Thus, from the pattern recognition perspective, we are dealing with a very complex and challenging problem, which up to date has not received too much attention in the state of the art due to the aforementioned difficulties. Most of the approaches found in the literature explore the relationship (mostly through regression, correlation analysis, or agreement metrics) between acoustic descriptors and the perceptual categories of a rating scale. Notwithstanding, there exist some methodologies dealing with the design of AVCA systems for the automatic evaluation of voice pathologies. For instance, features based on morphological properties of the modulation spectrum (MS), dimensionality reduction techniques, and a Gaussian Mixture Model (GMM) for classification are employed in Moro-Velázquez et al. (2015). Experiments are carried out in the MEEI corpus (Massachusetts Eye and Ear Infirmary, 1994) (assessed by 3 evaluators using the GRBAS scale), obtaining an accuracy of $81 \%$ for $G$, and $84 \%$ for $R$. In Ritchings et al. (2002), a system based on Artificial Neural Networks (ANN), spectral/cepstral and perturbation features has been used to provide assessments of laryngeal cancer to a series of patients evaluated according to a 7-rating scale of severity. The system provides an efficiency of $92 \%$. However, results are biased due to the lack of cross-validation, and having used frames of the same speakers for both training and testing purposes. Authors in Alpan et al. (2010) propose a system which classifies amongst normophonic, moderately hoarse or severely hoarse voices using signal-to-dysperiodicity ratios and Support Vector Machines (SVM). The methodology is tested on a corpus of connected speech, obtaining an efficiency of about 70\%. In Wang et al. (2016), 65 perturbation, spectral/cepstral and complexity features are used to predict $G$ in a dataset comprising 805 registers of sustained vowels (evaluated according to the GRB scale by 5 experts). Feature extraction techniques, and two types of classifiers are used for decision making, obtaining accuracies around $80 \%$. The work proposed in Stráník et al. (2014) describes a system that automatically classifies $B$ using running speech. The methodology is based on 92 features which are further reduced through feature selection. Using a dataset composed of 593 registers (assessed twice by 5 experts) a classification accuracy of $77 \%$ is obtained when the values of $B$ are computed as the mode of the 10 evaluations. The value of $B$ is also calculated as the mean value of the evaluations, and a regressor is used to assess performance. However, performance is only evaluated using the Pearson correlation $(\rho)$ between the values predicted by the regressor and the actual evaluations, reaching up a value of 0.92 . In Ma and Yiu (2006), registers of running speech belonging to 112 dysphonic and 41 normophonic patients (evaluated according to a 11-point scale of severity by 4 evaluators) are examined through aerodynamic, voice range profiles and acoustic perturbation metrics. Using dimensionality reduction techniques, a set of 4 features is finally selected and employed for classification using linear discriminant analysis. The attained efficiency is $67.3 \%$. Authors in Lee and Hahn (2010) extract higher-order statistics of linear prediction coding coefficients residuals. The objective is to assess $G$ automatically, in a corpus composed of 63 pathological recordings belonging to a Japanese dataset and 20 controls of a Korean dataset. By means of regression trees, a classification accuracy of $93 \%$ is obtained. Likewise, authors in Fredouille et al. (2009) employ a back-and-forth methodology based on cepstral analysis to optimise and refine an automatic assessment system providing decisions about $G$. Experiments are carried out in a dataset of 80 registers of short French texts, evaluated in consensus by three speech pathologists. The classification results using a GMM are of $78.8 \%$.

In view of the aforementioned, this paper presents an AVCA system, whose novelty with respect to the state of the art stands on the following aspects: (i) the development of a novel methodology based on multiple sets of characteristics, ordinal classification and Gaussian regression; (ii) experiments are performed on three training corpora, which have been assessed by the same speech therapist; (iii) the methodology seeks to generalise results performing experimentation in multiple corpora and following diverse procedures, in order to select a reduced set of robust and consistent features capable of dealing with most of the variability factors affecting this type of systems; (iv) the evaluation of the results is carried out following a cross-dataset strategy; ( $v$ ) in an attempt to account for the continuous nature that any assessment task should have, instead of a discrete output, the system 
provides a continuous value characterising the degree of pathology perceived in the input voice; (vi) in contrast to the current trend in the state of the art, instead of using the classification accuracy, the evaluation is carried out paying attention to the deviation of the labels assessed by the expert and those provided by the system, which is more in consonance with the inherent nature of the problem under study; (vii) the final system has been clinically evaluated by the same speech therapist who assessed the training corpora to test its transferability in a clinical environment.

This paper is organised as follows: Section 2 describes the corpora and experiments that are carried out; Section 3 presents the outcomes of the experiments; Section 4 introduce the discussions, whereas Section 5 presents the conclusions of this work.

\section{Experimental setup}

\subsection{Acoustic material}

Three corpora containing normophonic and pathological recordings are used as training corpora: Hospital Universitario Príncipe de Asturias (HUPA), Hospital Gregorio Marañón (GMar) and Saarbrücken (SVD) voice disorders corpora. Similarly, for the evaluation of results in cross-dataset experiments, two Spanish corpora of normophonic and dysphonic registers of sustained vowels are considered: the Hospital Doctor Negrín dataset (DN) and the Aplicación de las Tecnologías de la Información y las Comunicaciones (ATIC) corpora. All the corpora have been evaluated by the same speech therapist, a professional in voice analysis with more than 15 years of experience. A brief description of these corpora is given next:

\subsubsection{HUPA dataset}

Recorded at the Príncipe de Asturias hospital in Alcalá de Henares, Madrid, Spain Godino-Llorente et al. (2008). The dataset contains the sustained phonation of the vowel /a/ of 366 adult Spanish speakers (169 pathological and 197 normophonic). Registers have been recorded using the Kay Computerised Speech Lab Analysis station $4300 B$ with a sampling frequency of $50 \mathrm{kHz}$ and 16 bits of resolution. Pathological voices contain a wide variety of organic pathologies including nodules, polyps, oedemas, carcinomas, etc. The distribution of the $G, R$ and $B$ labels in the dataset is presented in Fig. 1(a).

\subsubsection{GMar dataset}

Composed of registers of Spanish speakers phonating the vowels /a/, / $\mathrm{i} /$ and $/ \mathrm{u} /$. The corpora has been recorded at the Gregorio Marañón Hospital, Madrid, Spain, using the MediVozCaptura system (Godino-Llorente et al., 2006) with a sampling frequency of $22050 \mathrm{~Hz}$ and 16 bits of resolution. The corpus is composed of 202 audio recordings, from which 95 are of normophonic and 107 of pathological speakers. For the purposes of this paper, only the vowel $/ a /$ is considered in the analysis. The distribution of the $G, R$ and $B$ labels is presented in Fig. 1(b).

\subsubsection{SVD dataset}

It holds a collection of audio registers from more than 2000 normophonic and pathological German speakers (Putzer and Barry, 2008; Saarbrüecken voice database). It was recorded by the Institut für Phonetik at Saarland University and the Phoniatry Section of the Caritas Clinic St. Theresia in Saarbrücken, Germany. The corpus comprises recordings of the sustained phonation of vowels /a/, /i/ and / $\mathrm{A} /$ uttered at normal, high and low pitch, as well as with rising-falling pitch. Besides, it incorporates recordings of the sentence Guten Morgen, wie geht es Ihnen? (Good morning, how are you?). Registers have been recorded using a sampling frequency of $50 \mathrm{kHz}$ and 16 bits of resolution. For the purposes of this paper only the vowel /a/ has been utilised, having defined a subset of the dataset after removing those registers with a low dynamic range or interferences. After this process, 1538 registers of speakers aged between 16 and 69 years are obtained (568 normophonic and 970 pathological). Fig. 1(c) depicts the distribution of the $G, R$ and $B$ labels in the dataset.

\subsection{4. $S V D p$ dataset}

An evaluation partition has also been defined by considering a randomly selected subset extracted from the SVD corpus, ensuring at least 50 registers labelled 0,50 labelled 1 , and so on. This partition is aimed for assessment purposes only. The distribution of the $G, R$ and $B$ labels for SVDp is presented in Fig. 1(d).

\subsubsection{DN dataset}

Recorded by Hospital Dr. Negrín Las Palmas de Gran Canaria, Spain. Contains 181 registers of Spanish speakers phonating the vowel /a/ (Alonso-Hernandez et al., 2001). The registers have been recorded at $22050 \mathrm{~Hz}$ and 16 bits of resolution. A partition of 130 registers has been randomly extracted and used for assessment purposes. The distribution of the $G, R$ and $B$ labels is depicted in Fig. 1(e).

\subsubsection{ATIC dataset}

Contains recordings of 79 Spanish speakers (58 dysphonic and 21 normophonic) phonating the vowel /a/ (ATIC). Pathological voices have been obtained from public and private Otorhinolaryngology services in Málaga, Spain, whereas normophonic speakers are teachers and students recruited at Málaga University. Registers have been recorded in quiet rooms under controlled conditions, digitised at 16 bits and $44100 \mathrm{~Hz}$. The voice samples have been perceptually labelled by three experts using the simplified GRB scale and a protocol promoting consensus among raters (more details about these procedures are described in ATIC). Besides this rating, an independent assessment is made by the speech therapist who evaluated the other corpora used in this work. This evaluation is presented in Fig. 1(f).

\subsection{Methodological setup}

Three main experiments are carried out in this paper. The first is devoted to find the most consistent set of features capable of predicting $G, B$ and $R$. To this end, several tests are performed in the training corpora: HUPA, GMar and SVD. With the lessons learnt during these three trials, a single AVCA system was built up and tested during the second experiment. The proposed AVCA system is assessed using three validation datasets: ATIC, DN and SVDp. Finally, in the third experiment, results are clinically evaluated by the speech therapist who has previously assessed all the datasets.

\subsubsection{Experiment 1. Design of the AVCA system}

Three trials are performed on each of the training corpora. The setup followed during each trial is presented in Fig. 2(a) and is described next:

I. Preprocessing: all registers are downsampled to $20 \mathrm{kHz}$ and maxnormalised, in such a manner that for a signal $s[t]$; its normalised version, $s_{\text {norm }}[t]$, is as follows:

$s_{\text {norm }}[t]=\frac{s[t]}{|\max \{s[t]\}|}$

where $\max \{\cdot\}$ is the maximum value in the register. A short time analysis is then employed to convert the audio register into frames according to a framing and windowing procedure. The length of the window is determined depending on the features that are extracted. Hamming windows are employed with different overlaps as to ensure that all the sets of characteristics contain the same number of frames.

II. Characterisation: this stage has the goal of extracting features capable of portraying the properties of normophonic and dysphonic conditions. Having a series of $P$ frames of speech, the idea is to obtain a matrix of characteristics $\mathfrak{X}=\left\{\vec{x}_{1}, \ldots, \vec{x}_{p} \ldots, \vec{x}_{P}\right\}$ : $\mathfrak{X} \in \mathbb{R}^{P \times d}$ where $\vec{x}_{p} \in \mathbb{R}^{d}$ is a vector of characteristics describing properties of the frame, and where $\mathfrak{X}$ is associated to a label $\ell$. The sets of characteristics that are considered in this paper are illustrated in Fig. 2(b) and are described next: 


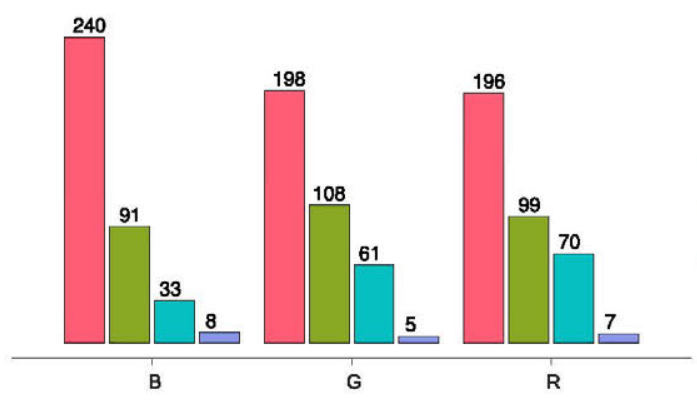

(a) HUPA database

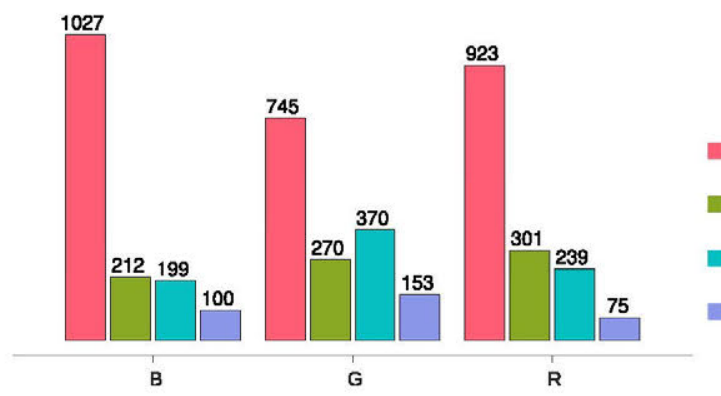

(c) SVD database

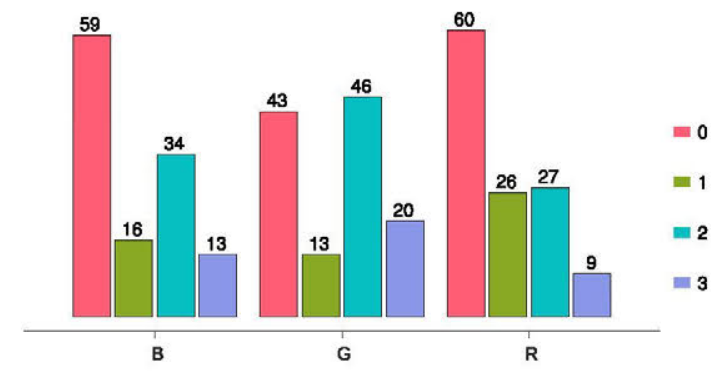

(e) DN database

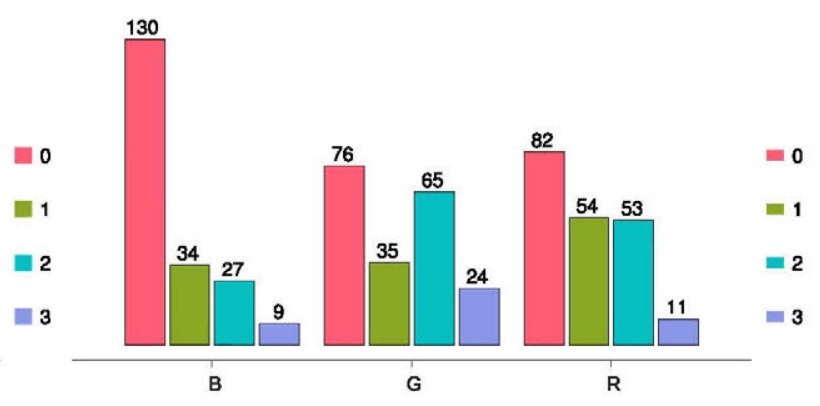

(b) GMar database

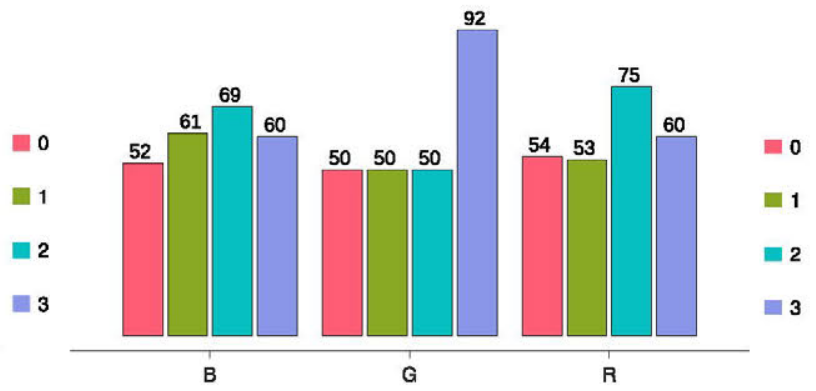

(d) SVDp partition

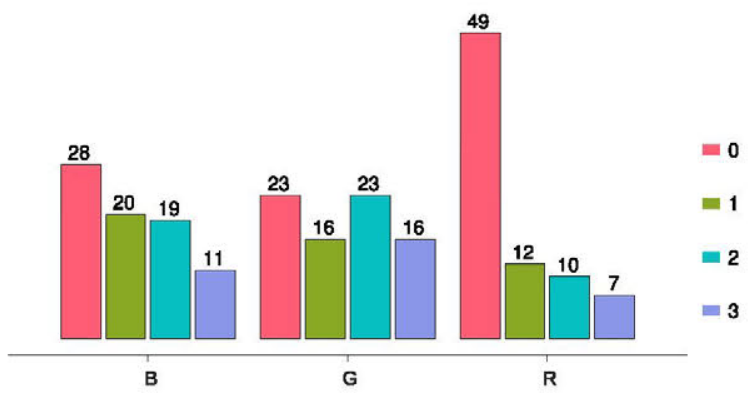

(f) ATIC database

Fig. 1. Classes associated to $G, R$ and $B$ for (a) HUPA, (b) GMar, (c) SVD, (d) SVDp, (e) DN, and (f) ATIC datasets.

- Perturbation features (Pert set): measure the presence of additive noise resulting from an incomplete glottal closure of the vocal folds; and the presence of modulation noise which is the result of irregularities in the movements of the vocal folds. Three perturbation metrics are considered: Normalised Noise Entropy (NNE) (Kasuya, 1986), Cepstral Harmonics-to-Noise Ratio (CHNR) (de Krom, 1993) and Glottal-to-Noise Excitation Ratio (GNE) (Michaelis et al., 1997). The length of the Hamming windows for this set is established to $40 \mathrm{~ms}$ as suggested in Arias-Londoño et al. (2011).

- Spectral and cepstral features (SpecCeps set): study the harmonic components of the voice. This set of features encloses Perceptual Linear Prediction coefficients (PLP) (Hermansky, 1990), Mel-Frequency Cepstral Coefficients (MFCC) (Arias-Londoño et al., 2011), Smoothed Cepstral Peak Prominence (CPPS) (Hillenbrand and Houde, 1996) and Low-toHigh Frequency Spectral Energy Ratio (LHr) (Awan et al., 2009). The length of the windows for the whole set is established to $40 \mathrm{~ms}$ as in Arias-Londoño et al. (2011). Likewise, PLP and MFCC coefficients are varied in the range of 10 to 20 with steps of 2 coefficients.

- Features based on modulation spectrum (MSs set): rely on the computation of the MS, that characterises the modulation and acoustic frequencies of input voices (Atlas and Shamma, 2003). The features considered in the set are: Modulation Spectrum Homogeneity (MSH), Cumulative Intersection Point (CIL), Rate of Points above Linear Average (RALA) and Modulation Spectrum Percentile (MSP)m, where the sub-index is referred to the percentile that is used, i.e. $\mathrm{MSP}_{25}, \mathrm{MSP}_{75}$ and $\mathrm{MSP}_{95}$ (Moro-Velázquez et al., 2015). For the characterisation of the set, Hamming windows of $180 \mathrm{~ms}$ are utilised as in Moro-Velázquez et al. (2015), whereas for the computation of the modulation spectrum 1024 modulation and 128 acoustic bands are employed.

- Complexity (Comp set): this family of parameters characterises the dynamic of the system and its structure. Several features are extracted, which are further grouped according to the properties they measure. Hence: (i) the first subset comprises dynamic invariants (Dyn subset) extracted from a reconstructed attractor such as the Correlation Dimension (D2), the Largest Lyapunov Exponent (LLE) (Kantz and Schreiber, 2004), and the Recurrence Period Density Entropy (RPDE) (Little et al., 2007); (ii) the second subset contain features which measure long-range correlations ( $L R$ subset), such as Hurst Exponent (He) and Detrended Fluctuation Analysis (DFA) (Peng et al., 1995; Little et al., 2007); (iii) the third subset includes regularity estimators (Reg subset) which are based on entropy-like quantifiers. It encompasses Approximate Entropy (ApEn) (Pincus, 1991), Sample 
Entropy (SampEn) (Richman and Moorman, 2000), Modified Sample Entropy (mSampEn) (Xie et al., 2008), Gaussian Kernel Sample Entropy (GSampEn) (Xu et al., 2005) and Fuzzy Entropy (FuzzyEn) (Chen et al., 2007); (iv) finally, the fourth subset includes entropy estimators (Ent subset) such as Permutation Entropy (PE) (Bandt, 2005; Zanin et al., 2012), Rényi Hidden Markov Model Entropy (rHMMEn) and Shannon Hidden Markov Model Entropy (sHMMEn) (AriasLondoño et al., 2011; Arias-Londoño and Godino-Llorente, 2015). In all these subsets Hamming windows of $55 \mathrm{~ms}$ length are used as in Arias-Londoño et al. (2011).

III. Decision making: let $\mathcal{X}=\left\{\mathfrak{X}_{1}, \ldots, \mathfrak{X}_{e}, \ldots, \mathfrak{X}_{E}\right\}$ be a training set of observations associated to a vector of labels $\vec{\ell}=\left\{\ell_{1}, \ldots, \ell_{e}\right.$, $\left.\ldots, \ell_{E}\right\}$, then the aim of the decision making procedure is to learn a mapping from the input observations to the labels. Two types of decision machines are studied in this paper. Each one differs with respect to the type of decisions they provide. In this regard, the first is based on an ordinal classification, which is aimed at predicting labels with an ordered arrangement, i.e, each label has a finite set of values, $\ell_{i} \in\left\{C_{1}, C_{2}, \ldots, C_{Q}\right\}$ and a natural label ordering exists, i. e., $C_{1} \prec C_{2} \cdots \prec C_{Q}$, where $\prec$ is an order relation operator. Two ordinal classifiers are employed (Gutierrez et al., 2016): Proportional Odd Model (POM) and Extreme Learning Machines with Ordered Partition (ELMOP). The other type of decision machine is based on regression, which outputs a continuous label in opposition to the discrete value given by classifiers. A single regressor is considered: Gaussian Mixture Regression (Stulp and Sigaud, 2015; Calinon, 2016). During experimentation, the number of hidden layers in the ELMOP algorithm is varied in the range of 5 to 95 with steps of 15 , whereas the number of Gaussians for the Gaussian regressor is varied in the set $\left\{2^{i}\right\}: i \in \mathbb{Z} ; 1 \leq i \leq 5$.

IV. Evaluation of results: metrics derived from the comparison between the predicted and the actual label are considered. These include the Mean Absolute Error (MAE), which is defined for an ordinal classification problem as the average deviation of the predicted class from the true class (Cardoso and Pinto, 2007; Cruz-Ramírez et al., 2014), which for $J$ instances is as follows:

$\operatorname{MAE}=\frac{1}{J} \sum_{j}\left|\mathcal{O}\left[\ell_{j}\right]-\mathcal{O}\left[\ell_{j}^{*}\right]\right|$

where $\ell_{j}$ are the actual and $\ell_{j}^{*}$ the predicted labels; and $\mathcal{O}[\cdot]$ is an operator indicating the position of the label in the ordinal rank (i. e., if a certain label $\ell_{j}$ can take up values $0,1,2$ and the label is 2, its position is 3). Similarly, Average Mean Absolute Error (AMAE) is defined as the macro averaged version of MAE (Baccianella et al., 2009):

$\mathrm{AMAE}=\frac{1}{Q} \sum_{q} \mathrm{MAE}_{q}$

where $\mathrm{MAE}_{j}$ is the MAE computed for instances having class $q$. The advantage of AMAE compared to MAE is its robustness to cope with data imbalance.

To deal with continuous labels, AMAE and MAE are modified by replacing the operator $\mathcal{O}[\cdot]$ for the value of the labels. Another measure that is usually employed in this context is the Root Mean Square Error (RMSE) which is computed as:

$\operatorname{RMSE}=\sqrt{\frac{\sum_{j}\left(\ell_{j}-\ell_{j}^{*}\right)^{2}}{J}}$

To analyse the interrelationship between features and labels, metrics of correlation such as the distance correlation $\left(\rho_{\mathcal{R}}\right)$ (Székely and Rizzo, 2014) and $\rho$ are utilised.

In addition and since assessments are subjective by nature, it is often necessary to compute statistics that provide information about the reliability of the ratings offered by the evaluators. This can be achieved using generalisability theory (O'Brian et al., 2003). In this manner, an ANOVA decomposition of the $k$ th true rating, $r_{i j k}$, (where the sub-index $i$ is related to the speaker, and $j$ is related to the rater) is as follows:

$r_{i j k}=\mu+S_{i}+R_{j}+S R_{i j}+e_{i j k}$

where $\mu$ is the grand-mean or the average score over the whole population of speakers and raters, $S_{i}$ is the difference between the true score for speaker $i$ and the grand mean, $R_{j}$ represents the average rating bias of rater $j$ relative to the average of all rates, $S R_{i j}$ models the interaction between rater and speaker, and $e_{i j k}$ incorporates the remaining sources of variation. Assuming independence of the different terms, the estimations of the variances can be performed by means of a two-way ANOVA model of random effects model, in such a manner that a decomposition of the variance terms $(\operatorname{var}(\cdot))$ is achieved as:

$\operatorname{var}(r)=\operatorname{var}(S)+\operatorname{var}(R)+\operatorname{var}(S R)+\operatorname{var}(e)$

Having this term, it is possible to compute a generalisability coefficient, which quantifies the consistency among the multiple measurements on a scale from 0 to 1 , and which is defined as (Webb et al., 2006):

$\mho=\frac{\operatorname{var}(S)}{\operatorname{var}(S)+\operatorname{var}(R) / N+\operatorname{var}(S R) / N+\operatorname{var}(e) / M N}$

where $M$ is the number of ratings given by the same rater, and $N$ is the total number of raters.

V. Dimensionality reduction: the objective of this procedure is to reduce the number of variables of the original space, in the search for a decrease in the computational burden, an improvement in the accuracy of the analyses, and the avoidance of problems such as the course of dimensionality (Cord and Cunningham, 2008). To this end, three dimensionality reduction methods are employed to rank features from the most to the least relevant. This analysis is performed independently for the HUPA, SVD and GMar datasets and for each one of the classes $(G, B$ and $R$ ) by means of three selection techniques (Brown et al., 2012): Maximal Information Maximisation (MIM), Minimal Redundancy Maximal Relevance (mRMR) and Joint Mutual Information (JMI).

VI. Selection of consistent characteristics: since the objective is to design a single system capable of generalising results, a scoring procedure is employed to select the best global set of features across training corpora and ranking algorithms. In this manner and with the results of the ranking techniques for a certain training dataset, the scoring procedure rewards the best features with a low score, while penalising the worst with a large value. The scores are then summed up across datasets and feature selection techniques. At the end, the features with the lowest scores are regarded as the most informative and consistent and are employed for further testing.

VII. Selection of most representative segments: a supermatrix is conformed by concatenating the most consistent features of the three training corpora. Since this matrix might contain uninformative segments, it is reasonable to select only the most representative utterances describing a certain class. This process resembles an "outlier removal" procedure which is applied as to find a more compact set of utterances. Due to the multidimensional nature of the features space, traditional outlier removal techniques cannot be applied. Instead, a GMM classifier is trained with one Gaussian for modelling the multidimensional supermatrix of features. Then, log-likelihood scores are computed for each row in the supermatrix. Next, scores are analysed by means of percentiles, removing utterances with scores below the median and conserving the remaining for further testing. 


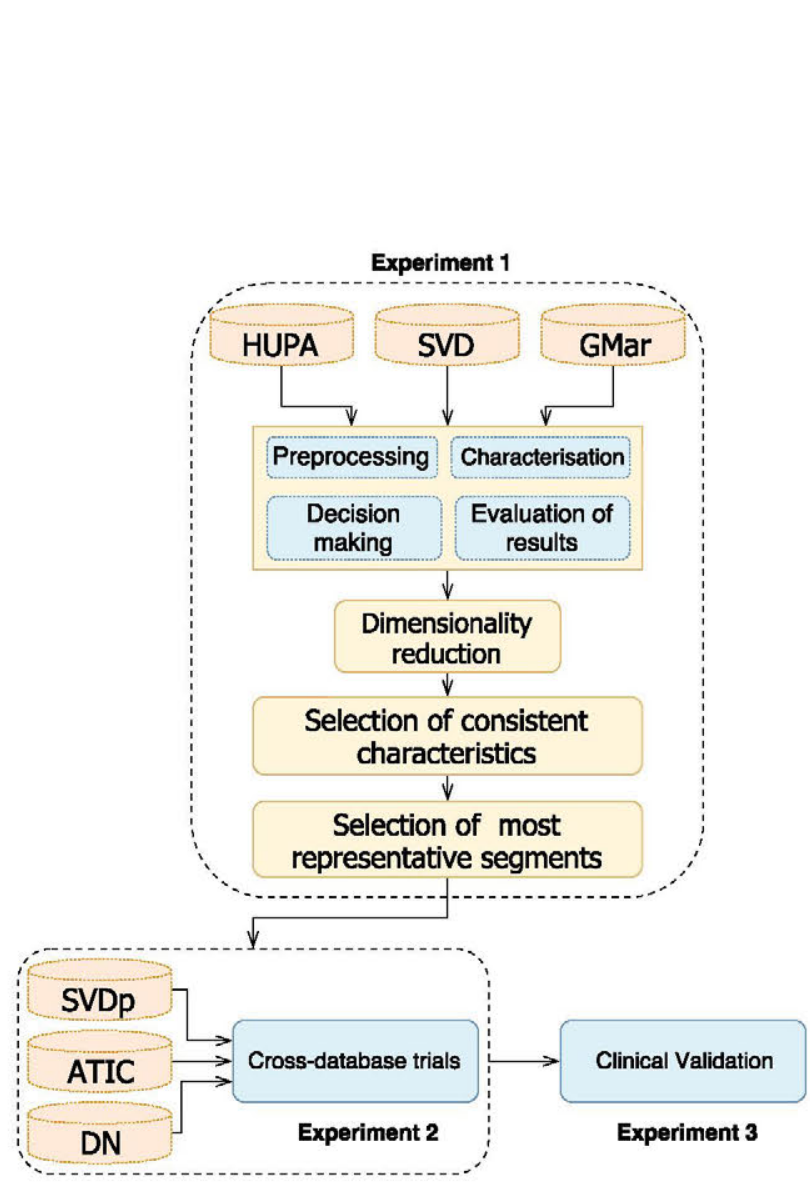

(a) Methodological stages of the AVCA system.

\begin{tabular}{|c|c|c|}
\hline Set & Subset & Features \\
\hline \multirow{3}{*}{ Pert } & - & HNR \\
\hline & - & NNE \\
\hline & - & GNE \\
\hline \multirow{4}{*}{ SpecCeps } & - & MFCC \\
\hline & - & CPPS \\
\hline & - & $\mathrm{LHr}$ \\
\hline & - & PLP \\
\hline \multirow{4}{*}{ MSs } & - & MSH \\
\hline & - & $\mathrm{CIL}$ \\
\hline & - & RALA \\
\hline & - & MSP \\
\hline \multirow{13}{*}{ Comp } & & LLE \\
\hline & Dyn & D2 \\
\hline & & $\mathrm{He}$ \\
\hline & & DFA \\
\hline & $\mathbf{L K}$ & RPDE \\
\hline & & ApEn \\
\hline & & SampEn \\
\hline & Reg & mSampEn \\
\hline & & GSampEn \\
\hline & & FuzzyEn \\
\hline & & $\mathrm{PE}$ \\
\hline & Ent & rHMMEn \\
\hline & & sHMMEn \\
\hline
\end{tabular}

(b) Feature sets and subsets used for characterisation.

Fig. 2. Methodology followed in this paper for the design of the AVCA system predicting $G, B$ and $R$.

\subsubsection{Experiment 2. Cross-dataset trials}

With the system previously trained, this experiment is devoted to test its performance in cross-dataset scenarios, using the following corpora: SVDp, ATIC and DN. The goal is to ensure that results are dataset independent.

\subsubsection{Experiment 3. Clinical validation}

Two assessment tasks are defined in this experiment. In the first, the evaluator is asked to provide a blind assessment of the registers after having re-imagined the GRB scale in a continuous fashion. A Matlab ${ }^{\circledR}$ application has been presented to the evaluator. This contains a slider which can be moved freely in a continuous range from 0 to 3 , and that includes ticks in the values $0,1,2$ and 3. During this task, the audio files of the SVDp and DN dataset are duplicated and randomised, thus obtaining a total of 484 registers for SVDp and 244 recordings for the DN dataset which are later employed to analyse the consistency of the specialist analysing the same voice material. In addition to that, the ATIC dataset has also been evaluated only once.

During the second task, the evaluator is enquired to supply a qualification about the accuracy of the labels predicted by the automatic system of the previous test. If any of the labels is too far from what it can be considered acceptable, the evaluator should move the slider to correct the evaluation. The errors are measured by means of RMSE and MAE.
Table 1

Experiment 1. Correlation between classes for the different datasets.

\begin{tabular}{|c|c|c|c|c|c|c|}
\hline & \multicolumn{2}{|l|}{$G-B$} & \multicolumn{2}{|l|}{$G-R$} & \multicolumn{2}{|l|}{$B-R$} \\
\hline & $\rho$ & $\rho_{\mathcal{R}}$ & $\rho$ & $\rho_{\mathfrak{R}}$ & $\rho$ & $\rho_{\mathcal{X}}$ \\
\hline HUPA & 0.74 & 0.73 & 0.81 & 0.79 & 0.71 & 0.70 \\
\hline SVD & 0.76 & 0.79 & 0.80 & 0.82 & 0.58 & 0.60 \\
\hline GMar & 0.67 & 0.67 & 0.86 & 0.84 & 0.53 & 0.49 \\
\hline
\end{tabular}

\section{Results}

\subsection{Experiment 1. Design of the generalist AVCA system}

Since all corpora have been assessed by the same speech therapist, a correlation analysis between pairs of classes is firstly provided in Table 1.

As observed, there are large correlations between $G-R$ and $G-B$. This is expected as $G$ is often considered a superclass encompassing $B$ and $R$. However, there are also large correlations (larger than 0.50 ) between $B-R$. This value is surprisingly high, specially for the HUPA dataset where $\rho_{\mathcal{R}}=0.70$ and SVD where $\rho_{\mathcal{R}}=0.60$. These correlations should have to be kept in mind to interpret the final results.

\subsubsection{Results for the HUPA dataset}

A correlation analysis between features and each one of the classes is reported in Table 2(a). Additionally, Table 2(b) presents the results of an ordinal classification that has been carried out to evaluate the performance of several sets of features in automatic assessment tasks. 
Table 2

Experiment 1. Performance and correlation metrics of the tested features and the $G . R$ and $B$ classes for the HUPA dataset.

\begin{tabular}{|c|c|c|c|c|c|c|c|}
\hline \multirow[b]{2}{*}{ Set } & \multirow[b]{2}{*}{ Features } & \multicolumn{2}{|l|}{$G$} & \multicolumn{2}{|l|}{$R$} & \multicolumn{2}{|l|}{ B } \\
\hline & & $\rho$ & $\rho_{\mathbb{R}}$ & $\rho$ & $\rho_{\mathcal{R}}$ & $\rho$ & $\rho_{\mathcal{R}}$ \\
\hline \multirow{3}{*}{ Pert } & NNE & -0.01 & 0.33 & -0.01 & 0.33 & -0.03 & 0.28 \\
\hline & CHNR & -0.52 & 0.48 & -0.51 & 0.47 & -0.42 & 0.40 \\
\hline & GNE & -0.55 & 0.54 & -0.49 & 0.48 & -0.38 & 0.40 \\
\hline \multirow{4}{*}{ SpecCeps } & PLP & - & 0.27 & - & 0.25 & - & 0.23 \\
\hline & MFCC & - & 0.23 & - & 0.22 & - & 0.22 \\
\hline & CPPS & -0.36 & 0.33 & -0.37 & 0.32 & -0.38 & 0.33 \\
\hline & $\mathrm{LHr}$ & -0.03 & 0.11 & -0.07 & 0.14 & -0.05 & 0.11 \\
\hline \multirow{6}{*}{ MSs } & MSH & 0.17 & 0.19 & 0.17 & 0.17 & 0.17 & 0.18 \\
\hline & CIL & -0.20 & 0.22 & -0.18 & 0.17 & -0.21 & 0.20 \\
\hline & RALA & -0.36 & 0.37 & -0.32 & 0.33 & -0.32 & 0.33 \\
\hline & $\mathrm{MSP}_{25}$ & -0.23 & 0.25 & -0.20 & 0.21 & -0.20 & 0.22 \\
\hline & $\mathrm{MSP}_{75}$ & -0.20 & 0.21 & -0.17 & 0.18 & -0.19 & 0.20 \\
\hline & $\mathrm{MSP}_{95}$ & -0.21 & 0.21 & -0.18 & 0.18 & -0.20 & 0.20 \\
\hline \multirow{13}{*}{ Comp } & $\mathrm{He}$ & 0.02 & 0.08 & 0.03 & 0.06 & -0.08 & 0.10 \\
\hline & DFA & 0.06 & 0.09 & 0.08 & 0.08 & -0.07 & 0.10 \\
\hline & D2 & 0.14 & 0.20 & 0.11 & 0.16 & 0.05 & 0.15 \\
\hline & LLE & 0.32 & 0.29 & 0.34 & 0.32 & 0.32 & 0.29 \\
\hline & RPDE & 0.13 & 0.21 & 0.21 & 0.25 & 0.16 & 0.19 \\
\hline & ApEn & -0.27 & 0.25 & -0.22 & 0.22 & -0.28 & 0.26 \\
\hline & SampEn & 0.11 & 0.17 & 0.06 & 0.14 & 0.07 & 0.14 \\
\hline & mSampEn & -0.08 & 0.13 & -0.13 & 0.14 & -0.16 & 0.16 \\
\hline & GSampEn & -0.03 & 0.07 & 0.03 & 0.08 & 0.03 & 0.06 \\
\hline & FuzzyEn & 0.08 & 0.13 & 0.07 & 0.13 & 0.09 & 0.12 \\
\hline & rHMMEn & 0.16 & 0.19 & 0.12 & 0.14 & 0.06 & 0.14 \\
\hline & sHMMEn & 0.15 & 0.17 & 0.12 & 0.13 & 0.06 & 0.13 \\
\hline & $\mathrm{PE}$ & 0.28 & 0.29 & 0.32 & 0.31 & 0.30 & 0.28 \\
\hline
\end{tabular}

\begin{tabular}{|c|c|c|c|c|c|c|c|c|}
\hline \multirow{2}{*}{ Set } & \multirow{2}{*}{ Subset } & \multirow{2}{*}{ Classifier } & \multicolumn{2}{|l|}{$G$} & \multicolumn{2}{|l|}{$R$} & \multicolumn{2}{|l|}{$B$} \\
\hline & & & MAE & AMAE & MAE & AMAE & MAE & AMAE \\
\hline \multirow{2}{*}{ Pert } & & POM & 0.39 & 0.37 & 0.47 & 0.43 & 0.41 & 0.34 \\
\hline & & ELMOP & 0.40 & 0.37 & 0.48 & 0.43 & 0.42 & 0.35 \\
\hline \multirow{6}{*}{ SpecCeps } & \multirow{2}{*}{ CPPS+LHr } & POM & 0.55 & 0.49 & 0.59 & 0.49 & 0.43 & 0.35 \\
\hline & & ELMOP & 0.58 & 0.44 & 0.63 & 0.45 & 0.45 & 0.34 \\
\hline & \multirow{2}{*}{ MFCC } & POM & 0.47 & 0.41 & 0.55 & 0.46 & 0.42 & 0.35 \\
\hline & & ELMOP & 0.46 & 0.41 & 0.53 & 0.44 & 0.40 & 0.32 \\
\hline & \multirow{2}{*}{ PLP } & POM & 0.45 & 0.39 & 0.54 & 0.47 & 0.41 & 0.33 \\
\hline & & ELMOP & 0.45 & 0.38 & 0.50 & 0.43 & 0.38 & 0.31 \\
\hline \multirow{2}{*}{ MSs } & & POM & 0.40 & 0.37 & 0.45 & 0.41 & 0.41 & 0.34 \\
\hline & & ELMOP & 0.41 & 0.37 & 0.45 & 0.38 & 0.40 & 0.33 \\
\hline \multirow{8}{*}{ Comp } & \multirow{2}{*}{ LR } & POM & 0.67 & 0.51 & 0.69 & 0.52 & 0.49 & 0.35 \\
\hline & & ELMOP & 0.66 & 0.51 & 0.73 & 0.55 & 0.51 & 0.37 \\
\hline & \multirow{2}{*}{ Dyn } & POM & 0.51 & 0.43 & 0.53 & 0.45 & 0.43 & 0.33 \\
\hline & & ELMOP & 0.48 & 0.40 & 0.55 & 0.45 & 0.42 & 0.33 \\
\hline & \multirow{2}{*}{ Reg } & POM & 0.54 & 0.48 & 0.61 & 0.51 & 0.48 & 0.38 \\
\hline & & ELMOP & 0.49 & 0.44 & 0.55 & 0.45 & 0.46 & 0.36 \\
\hline & \multirow{2}{*}{ Ent } & POM & 0.51 & 0.45 & 0.56 & 0.49 & 0.41 & 0.33 \\
\hline & & ELMOP & 0.45 & 0.40 & 0.48 & 0.41 & 0.44 & 0.36 \\
\hline
\end{tabular}

(b) Ordinal classification performance metrics.

(a) Correlation analysis of the tested features.

Results suggest that the Pert set has the highest interrelationship among all features, with characteristics such as GNE presenting correlation values superior to 0.38 no matter the class that is analysed. The Pert set also provides one of the best efficiencies when predicting $G$ (AMAE $=0.37$ ). Respecting the MSs set, it is noteworthy the consistent level of correlation of all features, with values up to 0.37 when using RALA. This set also provides some of the best outcomes in terms of classification performance when predicting $G(\mathrm{AMAE}=0.37)$ and $R$ (AMAE $=0.38$ ). Another well performing set is SpecCeps, with features such as CPPS having correlations above 0.33 for any class. Despite that, using CPPS in combination with LHr decreases results in the ordinal classification procedure. This might be expected due to the low correlation that $\mathrm{LHr}$ presents and that in some cases does not surpass 0.05 . PLP provides the best results in classification for $B$ with an $\mathrm{AMAE}=0.31$. Finally, and regarding the complexity features, it can be noticed that within the Comp set LLE provides the highest level of correlation (above 0.29 for any class), closely followed by PE (above 0.28 for any class) and ApEn (above 0.22 for any class). Notwithstanding the classification results are rather mediocre for this set.

\subsubsection{Results for the SVD dataset}

A correlation analysis between the tested features and the $G, R$ and $B$ is shown in Table 3(a). Likewise, ordinal classification results are in Table 3(b).

Similarly to the previous trial, the best results are achieved when using the Pert set (with correlation values above 0.53 using CHNR and above 0.44 using GNE) and classification results that are consistently higher than in the remaining sets (AMAE for $G=0.60, B=0.74$ and $R=0.78$ ). Considering the MSs set, RALA is once again the best correlated characteristic, although, being closely followed by the remaining (in all cases always above 0.4) but for MSH (above 0.18). The performance of the MSs set is one of the highest among the trial (AMAE ranges from 0.69 in $G$ to 0.84 in $R$ ). With regard to SpecCeps,
CPPS and PLP present the highest correlation values within the set (always superior to 0.31 for all classes), but its performance in ordinal classification is moderate (specially for CPPS $+\mathrm{LHr}$ where AMAE ranges from 0.92 to 1.13). Respecting the Comp set, PE and LLE achieve levels of correlation above 0.41 for all classes. In contrast, the regularity features are more modestly correlated. In terms of ordinal classification performance, Ent (where PE is contained) presents some of the most competitive outcomes next to Dyn (AMAE ranging from 0.72 to 0.97 for these two sets).

\subsubsection{Results for the GMar dataset}

The correlation analysis between the tested features and the $G, R$ and $B$ classes is presented in Table 4(a), whereas the results for the ordinal classification are introduced in Table 4(b).

As in the previous two trials, the largest correlation values are generally obtained with the Pert set, having $\rho_{\mathcal{R}}=0.39$ when using GNE and CHNR. This set also achieves good performance in ordinal classification tasks, specially for $G(\mathrm{AMAE}=0.50)$ and $R$ ( $\mathrm{AMAE}=$ 0.51). Relating to the MSs set, correlation values are amongst the largest within the trial, ranging from 0.44 with RALA to 0.11 with MSH. In relation to the SpecCeps set, correlations are above 0.23 in all cases, but with CPPS and LHr providing the highest values. Notwithstanding, the best classification performance is obtained with the PLP features, specially for predicting $B($ AMAE $=0.32$ ). With reference to the Comp set, there is a modest classification performance in each one of the subsets.

\subsubsection{General comments}

Results are fairly consistent when using the three corpora. From the presented outcomes it can be inferred that the Pert set provides the best correlation among sets, as ascertained by the results of the three trials. Likewise, both MSs and SpecCeps also present some of the largest correlation values and a homogeneous behaviour among 
Table 3

Experiment 1. Performance and correlation metrics of the tested features and the $G . R$ and $B$ classes for the SVD dataset.

\begin{tabular}{|c|c|c|c|c|c|c|c|}
\hline \multirow[b]{2}{*}{ Set } & \multirow[b]{2}{*}{ Features } & \multicolumn{2}{|l|}{$G$} & \multicolumn{2}{|l|}{$R$} & \multicolumn{2}{|l|}{ B } \\
\hline & & $\rho$ & $\rho_{\mathcal{R}}$ & $\rho$ & $\rho_{\mathcal{R}}$ & $\rho$ & $\rho_{\mathcal{R}}$ \\
\hline \multirow{3}{*}{ Pert } & NNE & 0.02 & 0.45 & 0.04 & 0.43 & 0.02 & 0.43 \\
\hline & CHNR & -0.62 & 0.58 & -0.55 & 0.53 & -0.58 & 0.55 \\
\hline & GNE & -0.57 & 0.54 & -0.46 & 0.44 & -0.59 & 0.57 \\
\hline \multirow{4}{*}{ SpecCeps } & PLP & - & 0.31 & - & 0.31 & - & 0.30 \\
\hline & MFCC & - & 0.28 & - & 0.20 & - & 0.25 \\
\hline & CPPS & -0.34 & 0.32 & -0.32 & 0.30 & -0.34 & 0.32 \\
\hline & $\mathrm{LHr}$ & -0.16 & 0.23 & -0.20 & 0.27 & -0.20 & 0.26 \\
\hline \multirow{6}{*}{ MSs } & MSH & -0.24 & 0.23 & -0.19 & 0.18 & -0.23 & 0.23 \\
\hline & CIL & 0.49 & 0.45 & 0.47 & 0.44 & 0.48 & 0.44 \\
\hline & RALA & 0.55 & 0.51 & 0.51 & 0.48 & 0.54 & 0.51 \\
\hline & $\mathrm{MSP}_{25}$ & 0.45 & 0.42 & 0.42 & 0.40 & 0.47 & 0.43 \\
\hline & $\mathrm{MSP}_{75}$ & 0.48 & 0.44 & 0.45 & 0.43 & 0.48 & 0.44 \\
\hline & $\mathrm{MSP}_{95}$ & 0.52 & 0.48 & 0.50 & 0.48 & 0.55 & 0.50 \\
\hline \multirow{13}{*}{ Comp } & $\mathrm{He}$ & 0.29 & 0.31 & 0.21 & 0.22 & 0.24 & 0.28 \\
\hline & DFA & 0.32 & 0.33 & 0.24 & 0.24 & 0.26 & 0.28 \\
\hline & D2 & 0.01 & 0.30 & 0.00 & 0.22 & 0.01 & 0.29 \\
\hline & LLE & 0.44 & 0.41 & 0.42 & 0.41 & 0.47 & 0.42 \\
\hline & RPDE & 0.30 & 0.33 & 0.39 & 0.40 & 0.27 & 0.30 \\
\hline & ApEn & -0.18 & 0.17 & -0.12 & 0.13 & -0.22 & 0.20 \\
\hline & SampEn & 0.06 & 0.14 & 0.05 & 0.09 & 0.09 & 0.14 \\
\hline & mSampEn & -0.11 & 0.12 & -0.16 & 0.15 & -0.07 & 0.09 \\
\hline & GSampEn & -0.02 & 0.13 & 0.05 & 0.12 & -0.02 & 0.13 \\
\hline & FuzzyEn & -0.02 & 0.16 & 0.05 & 0.09 & 0.03 & 0.14 \\
\hline & rHMMEn & 0.12 & 0.17 & 0.03 & 0.11 & 0.13 & 0.16 \\
\hline & sHMMEn & 0.14 & 0.17 & 0.08 & 0.13 & 0.15 & 0.17 \\
\hline & $\mathrm{PE}$ & 0.49 & 0.43 & 0.53 & 0.48 & 0.48 & 0.42 \\
\hline
\end{tabular}

\begin{tabular}{|c|c|c|c|c|c|c|c|c|}
\hline \multirow{2}{*}{ Set } & \multirow{2}{*}{ Subset } & \multirow{2}{*}{ Classifier } & \multicolumn{2}{|l|}{$G$} & \multicolumn{2}{|l|}{$R$} & \multicolumn{2}{|l|}{$B$} \\
\hline & & & MAE & AMAE & MAE & AMAE & MAE & AMAE \\
\hline \multirow{2}{*}{ Pert } & & POM & 0.47 & 0.60 & 0.43 & 0.78 & 0.35 & 0.74 \\
\hline & & ELMOP & 0.47 & 0.59 & 0.44 & 0.82 & 0.36 & 0.74 \\
\hline \multirow{6}{*}{ SpecCeps } & \multirow{2}{*}{$\mathrm{CPPS}+\mathrm{LHr}$} & POM & 0.73 & 0.92 & 0.54 & 1.13 & 0.49 & 1.18 \\
\hline & & ELMOP & 0.79 & 1.03 & 0.57 & 1.27 & 0.51 & 1.19 \\
\hline & \multirow{2}{*}{ MFCC } & POM & 0.64 & 0.80 & 0.51 & 0.95 & 0.45 & 0.95 \\
\hline & & ELMOP & 0.58 & 0.73 & 0.51 & 0.96 & 0.42 & 0.89 \\
\hline & \multirow{2}{*}{ PLP } & POM & 0.57 & 0.70 & 0.46 & 0.83 & 0.39 & 0.78 \\
\hline & & ELMOP & 0.54 & 0.68 & 0.46 & 0.85 & 0.40 & 0.82 \\
\hline \multirow{2}{*}{ MSs } & & POM & 0.57 & 0.69 & 0.46 & 0.84 & 0.38 & 0.78 \\
\hline & & ELMOP & 0.58 & 0.74 & 0.47 & 0.94 & 0.41 & 0.91 \\
\hline \multirow{8}{*}{ Comp } & \multirow{2}{*}{ LR } & POM & 0.75 & 1.02 & 0.63 & 1.33 & 0.57 & 1.38 \\
\hline & & ELMOP & 0.74 & 1.04 & 0.61 & 1.29 & 0.55 & 1.31 \\
\hline & \multirow{2}{*}{ Dyn } & POM & 0.61 & 0.75 & 0.48 & 0.91 & 0.44 & 0.97 \\
\hline & & ELMOP & 0.57 & 0.71 & 0.46 & 0.87 & 0.42 & 0.92 \\
\hline & \multirow{2}{*}{ Reg } & POM & 0.87 & 1.06 & 0.61 & 1.32 & 0.55 & 1.35 \\
\hline & & ELMOP & 0.64 & 0.84 & 0.60 & 1.31 & 0.51 & 1.19 \\
\hline & \multirow{2}{*}{ Ent } & POM & 0.61 & 0.72 & 0.46 & 0.82 & 0.43 & 0.92 \\
\hline & & ELMOP & 0.55 & 0.69 & 0.46 & 0.87 & 0.42 & 0.89 \\
\hline
\end{tabular}

(b) Ordinal classification performance metrics.

(a) Correlation analysis of the tested features.

Table 4

Experiment 1. Performance and correlation metrics of the tested features and the $G . R$ and $B$ classes for the GMar dataset.

\begin{tabular}{|c|c|c|c|c|c|c|c|}
\hline \multirow[b]{2}{*}{ Set } & \multirow[b]{2}{*}{ Features } & \multicolumn{2}{|l|}{$G$} & \multicolumn{2}{|l|}{$R$} & \multicolumn{2}{|l|}{$B$} \\
\hline & & $\rho$ & $\rho_{\mathcal{R}}$ & $\rho$ & $\rho_{\mathcal{K}}$ & $\rho$ & $\rho_{\mathfrak{R}}$ \\
\hline \multirow{3}{*}{ Pert } & NNE & -0.02 & 0.23 & 0.02 & 0.21 & -0.04 & 0.20 \\
\hline & CHNR & -0.47 & 0.45 & -0.42 & 0.40 & -0.39 & 0.39 \\
\hline & GNE & -0.46 & 0.46 & -0.43 & 0.43 & -0.40 & 0.41 \\
\hline \multirow{4}{*}{ SpecCeps } & PLP & - & 0.32 & - & 0.29 & - & 0.29 \\
\hline & MFCC & - & 0.28 & - & 0.25 & - & 0.29 \\
\hline & CPPS & -0.39 & 0.36 & -0.25 & 0.23 & -0.35 & 0.32 \\
\hline & $\mathrm{LHr}$ & -0.29 & 0.35 & -0.24 & 0.30 & -0.26 & 0.32 \\
\hline \multirow{6}{*}{ MSs } & MSH & -0.13 & 0.13 & -0.12 & 0.12 & -0.11 & 0.12 \\
\hline & CIL & 0.34 & 0.31 & 0.27 & 0.24 & 0.32 & 0.30 \\
\hline & RALA & 0.44 & 0.44 & 0.41 & 0.42 & 0.38 & 0.39 \\
\hline & $\mathrm{MSP}_{25}$ & 0.36 & 0.33 & 0.25 & 0.24 & 0.35 & 0.33 \\
\hline & $\mathrm{MSP}_{75}$ & 0.34 & 0.31 & 0.24 & 0.22 & 0.33 & 0.30 \\
\hline & $\mathrm{MSP}_{95}$ & 0.38 & 0.34 & 0.28 & 0.25 & 0.39 & 0.36 \\
\hline \multirow{13}{*}{ Comp } & $\mathrm{He}$ & 0.06 & 0.17 & 0.16 & 0.21 & 0.02 & 0.14 \\
\hline & DFA & 0.03 & 0.12 & 0.09 & 0.13 & 0.00 & 0.10 \\
\hline & $\mathrm{D} 2$ & 0.06 & 0.20 & 0.07 & 0.20 & 0.05 & 0.18 \\
\hline & LLE & 0.31 & 0.27 & 0.28 & 0.25 & 0.36 & 0.31 \\
\hline & RPDE & 0.09 & 0.18 & 0.10 & 0.18 & 0.10 & 0.15 \\
\hline & ApEn & -0.24 & 0.21 & -0.18 & 0.16 & -0.25 & 0.22 \\
\hline & SampEn & 0.07 & 0.13 & 0.04 & 0.10 & 0.09 & 0.16 \\
\hline & mSampEn & -0.14 & 0.15 & -0.09 & 0.10 & -0.14 & 0.13 \\
\hline & GSampEn & -0.05 & 0.10 & -0.03 & 0.08 & -0.05 & 0.09 \\
\hline & FuzzyEn & 0.06 & 0.10 & 0.02 & 0.09 & 0.15 & 0.13 \\
\hline & rHMMEn & 0.08 & 0.18 & 0.07 & 0.17 & 0.08 & 0.16 \\
\hline & sHMMEn & 0.11 & 0.17 & 0.08 & 0.16 & 0.11 & 0.16 \\
\hline & $\mathrm{PE}$ & 0.29 & 0.30 & 0.23 & 0.24 & 0.30 & 0.28 \\
\hline
\end{tabular}

\begin{tabular}{|c|c|c|c|c|c|c|c|c|}
\hline \multirow{2}{*}{ Set } & \multirow{2}{*}{ Subset } & \multirow{2}{*}{ Classifier } & \multicolumn{2}{|l|}{$G$} & \multicolumn{2}{|l|}{$R$} & \multicolumn{2}{|l|}{$B$} \\
\hline & & & MAE & AMAE & MAE & AMAE & MAE & AMAE \\
\hline \multirow{2}{*}{ Pert } & & POM & 0.66 & 0.52 & 0.65 & 0.55 & 0.49 & 0.36 \\
\hline & & ELMOP & 0.64 & 0.50 & 0.62 & 0.51 & 0.54 & 0.34 \\
\hline \multirow{6}{*}{ SpecCeps } & \multirow{2}{*}{$\mathrm{CPPS}+\mathrm{LHr}$} & POM & 0.76 & 0.65 & 0.76 & 0.67 & 0.50 & 0.36 \\
\hline & & ELMOP & 0.97 & 0.83 & 0.76 & 0.69 & 0.58 & 0.37 \\
\hline & \multirow{2}{*}{ MFCC } & POM & 0.79 & 0.61 & 0.73 & 0.59 & 0.50 & 0.35 \\
\hline & & ELMOP & 0.68 & 0.50 & 0.79 & 0.62 & 0.47 & 0.35 \\
\hline & \multirow{2}{*}{ PLP } & POM & 0.72 & 0.59 & 0.73 & 0.60 & 0.46 & 0.34 \\
\hline & & ELMOP & 0.70 & 0.51 & 0.73 & 0.55 & 0.45 & 0.32 \\
\hline \multirow{2}{*}{ MSs } & & POM & 0.71 & 0.55 & 0.70 & 0.61 & 0.50 & 0.34 \\
\hline & & ELMOP & 0.79 & 0.66 & 0.71 & 0.66 & 0.53 & 0.34 \\
\hline \multirow{8}{*}{ Comp } & \multirow{2}{*}{ LR } & POM & 0.96 & 0.82 & 0.74 & 0.64 & 0.58 & 0.35 \\
\hline & & ELMOP & 0.93 & 0.71 & 0.77 & 0.66 & 0.59 & 0.36 \\
\hline & \multirow{2}{*}{ DynInv } & POM & 0.82 & 0.66 & 0.70 & 0.60 & 0.51 & 0.33 \\
\hline & & ELMOP & 0.82 & 0.63 & 0.72 & 0.58 & 0.50 & 0.33 \\
\hline & \multirow{2}{*}{$\operatorname{Reg}$} & POM & 0.83 & 0.67 & 0.72 & 0.64 & 0.53 & 0.34 \\
\hline & & ELMOP & 0.75 & 0.63 & 0.70 & 0.62 & 0.51 & 0.34 \\
\hline & \multirow{2}{*}{ EntAtt } & POM & 0.86 & 0.73 & 0.76 & 0.67 & 0.53 & 0.34 \\
\hline & & ELMOP & 0.66 & 0.55 & 0.65 & 0.55 & 0.49 & 0.34 \\
\hline
\end{tabular}

(b) Ordinal classification performance metrics.

(a) Correlation analysis of the tested features. 
Table 5

Experiment 1. Top-10 ranked features, after considering the three feature selection techniques and the scoring procedure.

\begin{tabular}{lll}
\hline$G$ & $B$ & $R$ \\
\hline CHNR & CHNR & PE \\
PE & GNE & GNE \\
GNE & PE & RALA \\
RALA & PLP(2) & CHNR \\
PLP(3) & RALA & PLP(3) \\
PLP(2) & MSP $_{95}$ & NNE \\
MSP $_{75}$ & PLP(3) & MSP \\
NNE & NNE & CPPS \\
CPPS & CPPS & LLE \\
CIL & LLE & MFCC(16) \\
\hline
\end{tabular}

trials. By contrast, Comp provides fluctuating outcomes. In terms of individual characteristics, a common pattern of high correlation and good performance is generally observed with RALA, CPPS, GNE and CHNR. Nonetheless, other well-performing/well-correlated characteristics include LLE, ApEn and PE. An interesting observation from the experiment is that features tend to perform equally well no matter the class that is analysed. This might contradict the reasoning of some features correlating better with some classes as they are expected to measure a certain phenomenon of pathological voice production. For instance, we would expect characteristics measuring modulation noise (such as RPDE) to have a higher correspondence to the $R$ class, whereas those indicating the presence of additive noise (such as perturbation measures) to be more interrelated to $B$. This is because additive noise tends to be associated to the perception of breathiness due to turbulence, whereas modulation noise tends to be interrelated to the roughness perception. However, this is not the case, and in contrast a subset of characteristics dominate over $G, B$ and $R$. It is important to remark that features have been evaluated individually, or grouped into subsets according to the domain they have been computed. Thus, an analysis that assesses the complementarity of features - even if calculated in different domains - in the search of a combination that most reliably characterises vocal quality, should be performed.

To provide further evidences about the performance of each tested sets of characteristics, three dimensionality reduction algorithms (MIM, mRMR and JMI) are applied. Thus, for each dataset, a supermatrix is created by concatenating all sets of features. The aim is to rank the features within each set from the most to the least relevant (according to the filter algorithms). To select the most consistent characteristic and generalise the ranking results disregarding the effect of the dataset and the filter algorithm, a scoring procedure is performed as explained in the previous section. The outcomes after following such process are referred in Table 5. It can be noticed that the most informative features (for all classes) are GNE, PE, RALA and CHNR, with other features such as some PLP, NNE or MSP $_{95}$ performing well.

Intensive testing has demonstrated the good performance provided by a set conformed by RALA, GNE and CHNR exclusively, in contraposition to other combinations. Therefore, only these three features were used to build up the system with the information of the HUPA, GMar and SVD datasets. To concentrate all the information of the registers into a single data-point, thus avoiding dispersion in the feature space, a per-file statistic is computed for each one of the registers as the average value of the feature matrix $\mathfrak{X}$. As proven later after extensive experimentation, this averaging process has been useful for improving results in automatic assessment tasks. As a matter of example and to illustrate the dispersion phenomenon, Fig. 3 introduces the CPPS contour formed after plotting all the frames of two voice recordings, one labelled $G=0$ (blue) and the other $G=2$ (red). As observed, the register with label 0 presents a very consistent behaviour, with a high CPPS value which is typical of voices not affected by the presence of turbulent noise. By contrast, the register labelled 2 contains a widespread distribution of points with continuous drops and rises. Indeed, in some utterances the values are practically similar to those attained by the register labelled

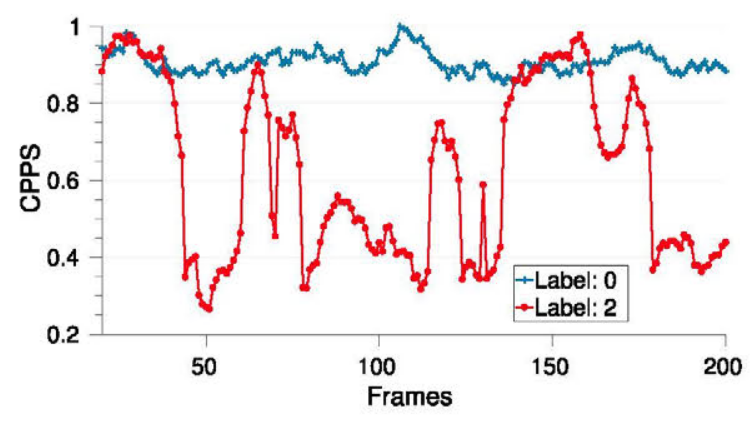

Fig. 3. Contour formed after plotting the CPPS of two voices belonging to the HUPA dataset. The graphic in red is for a voice labelled with $G=2$, whereas the one in blue is labelled $G=0$.

0 . This indicates that even in pathological conditions, there exist frames resembling a normophonic behaviour.

The next stage in the design methodology is the construction of a supermatrix, for each one of the classes, which is built up by concatenating the previously obtained per-file metrics of the training corpora. A further processing is performed to select the most representative rows in the supermatrix, resembling an outlier-removal procedure but accounting for the multidimensional nature of the supermatrix. To this end, each label $(1,2$ or 3$)$ within each class $(G, B$ or $R$ ) is modelled using a GMM trained with 1 Gaussian. Then, the log-likelihood between each data point (row in the supermatrix) and the model to which it belongs is computed. The resulting score measures how likely is the data-point to be generated by the model. Boxplots of the scores are then drawn as they provide information about the distribution of their values. Only rows whose scores are above a certain threshold are kept for further testing, hence providing a more compact representation of the labels under study. After some testing using different threshold values, the median or 50th percentile is used due to the good results that are obtained.

\subsection{Experiment 2. Cross-dataset trials}

As previously explained, the present experiment is devoted to assess results in cross-dataset scenarios to ensure the generalisation of the results. In order to evaluate the performance of the system in the following trials, the confusion matrices resulting from the ordinal classification procedures are depicted. Moreover, and for comparing these results to a certain baseline, an artificial confusion matrix is constructed by assuming the existence of four Probability Mass Functions (pmf) for each label. Each pmf is designed optimistically to ensure a large probability of occurrence for the label itself and its neighbour labels (0.9), and low elsewhere (the remaining 0.10). The graphic illustrating the pmf for each one of the labels is presented in Fig. 4. As a matter of example, the pmf for label 0 assumes that a sample extracted randomly will produce, with a probability of 0.7 , a value labelled as 0 ; with a probability of 0.2 a value labelled 1 ; and with a probability of 0.05 a value that would be either 2 or 3 . Values of the confusion matrices are randomly sampled from these pmf by means of an inverse transform sampling procedure (Sugiyama, 2016). The number of samples taken with the artificial model is the same as the number of available instances of the target label.

In addition, a weighted version of AMAE is used to measure the influence of the errors around the diagonal of the confusion matrices. The measure is a modification of the statistics extracted from the weighted confusion matrix as discussed in Rossiter (2014), but adapted to the context of an ordinal classification. In this respect, the classical AMAE assumes that the weight that is usually associated to errors around the diagonal is equal to 1 . The weighted-AMAE is a generalisation that allows this value to be varied in the range 0 -where an error around the diagonal is regarded as accurate - to 1 -the usual case -. 


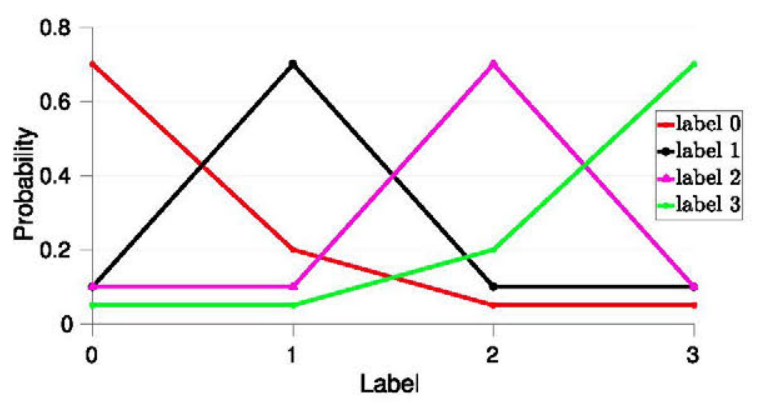

Fig. 4. pmf for the labels $0,1,2$ or 3 . These are used in the design of the random samples generator.

\subsubsection{Results for $S V D p$}

POM and ELMOP ordinal classifiers are trained for testing out the ability of the selected features in predicting $G, B$ and $R$. The outcomes of the ordinal classification procedure are introduced in Table 6(a). Similarly, the confusion matrices of the technique rendering the best results, along with its weighted-AMAE representation, are presented in Fig. 5.

As observed in Table 6(a), the POM algorithm achieves the best classification results in terms of AMAE for all classes. In spite of that, the outcomes provided by both classifiers are close to each other suggesting consistency of the selected features and of the techniques. Revisiting the results of the confusion matrices in Fig. 5, large errors are encountered between the label 1 and its neighbour label 0 . In terms of sensitivity, label 1 ranges from $18 \%$ to $34 \%$ in any of the tested classes. Similarly, the label 2 presents - for all classes - large errors, specially due to confusions with the neighbour label 3. By contrast, 0 and 3 are very well represented. In particular, label 0 has sensitivity values ranging from $74 \%$ to $90.4 \%$, whereas 3 varies from $43.3 \%$ to $72.8 \%$. For class $G$, the confusion matrix resembles the matrix of the artificial system. This is not the case for the remaining classes. One interesting observation is that most of the errors are distributed around the diagonal, i.e, errors arise in the neighbourhood of the target label. Indeed, and as observed in the graphics considering the weightedAMAE (centre-right plots in Fig. 5), by setting the weights to 0 -and thus regarding the errors around the diagonal as positive outcomes- AMAE is decreased drastically to around 0.16 for $G$ and $B$, and to about 0.20 for $R$.

Now, and since the assessment of voice quality should consider the whole spectrum of possible evaluations ranging from normophonia to severe dysphonia, the use of a discrete scale might not be optimal. Hence, and to acknowledge for the continuous nature of the problem, a Gaussian regressor is tested out. The decisions given by the regressor are then compared to the original $G, R, B$ labels to compute error metrics. Following this approach, Table 7 (a) presents the RMSE and MAE values when using the SVDp corpus. In addition, raster plots representing the deviation between predicted and target labels are the rightmost figures in Fig. 5. In particular, the raster plot depicts the occurrences of the predicted label. It is expected that the closer the predictions to the targets, the more compact the distribution of points around the actual label. Analysing the results obtained for $G$, it can be inferred that the label 3 is well represented as given by data-points centred around the value 3 . Even though there are certain values that deviate, the performance in general terms is good (RMSE $=0.69$ ). The behaviour of label 2 is acceptable, having some confusions with the label 3. Regarding $B$, a large overlap between labels 2 and 3, and between labels 0 and 1 is noticed. The outcomes for class $R$-just as in $B$ - reveal a large overlap between labels 0 and 1 , and between 2 and 3. Regarding the outcomes of Table 7(a), $G$ presents the best results as expressed by RMSE and MAE ( 0.69 and 0.51 respectively). Large errors are achieved for the classes $R$ and $B$ (RMSE $=0.76$ and RMSE $=0.72$ respectively), which might be due to the significant overlap occurring between labels $2-3$ as observed in the raster plots.

\subsubsection{Results for the DN partition}

The results of the ordinal classification procedure are in Table 6(b). Similarly, the confusion matrices of the classification method providing the best results are presented in Fig. 6 .

The outcomes indicate an acceptable performance for $G$ and $B$ (AMAE of 0.49 and 0.48 respectively), and a decreased efficiency for $R$ (AMAE=0.71). The ELMOP technique tends to outperform POM but in the $B$ case. Revisiting the confusion matrices in Fig. 6 , it can be observed that for the $G$ class, most of the errors occurs in the neighbour label, whereas errors are more widespread when $B$ and $R$ are considered. The label 1 is the one with the worst results among classes, with a sensitivity ranging from $7.7 \%$ for $G$, to $26.9 \%$ for $B$. The label 0 is well modelled, achieving a sensitivity of $83.7 \%$ for $G$, of $78 \%$ for $B$ and of $60 \%$ for $R$. Similarly the label 3 achieves a sensitivity ranging from $65 \%$ for $G$ to $100 \%$ for B. As in the trial involving SVDp, and as ascertained by the graphic of the weighted-AMAE, accepting errors in the diagonals decreases AMAE to about 0.09 for $G$, to 0.16 for $B$ and to 0.34 for $R$.

Table 7(b) presents the RMSE and MAE values obtained using the DN partition. Similarly, raster plots representing the deviation between predicted and actual labels are presented as the rightmost plots in Fig. 6. The outcomes of the Gaussian regressor indicate that the system predicts successfully $G$ and $B$ (RMSE $=0.69$ in both cases). However, results for $R$ are not nearly as good (RMSE $=0.96$ ). Interestingly, the raster plots indicate certain consistency in the results. However, label 3 tended to be centred towards $2-2.5$ instead of getting closer to 3 . Additionally, the worst performance is provided by $R$ with a large overlap between labels 0,1 and 2 . It is worth noting that unlike the previous trial, where at least 50 labels of each type $(0,1,2,3)$ are ensured, in this particular test there are some cases with no more than 9 observations.

\subsubsection{Results for the ATIC dataset}

The results of the ordinal classification procedure using the ATIC dataset are introduced in Table 6(c). Similarly, the confusion matrices of the classification method providing the best results are in Fig. 7.

Results of Table $6(\mathrm{c})$ indicate that POM outperforms ELMOP. In general the error rates in terms of AMAE are larger than in the two previous trials, with $B$ providing the best results $(0.60)$, and $R$ the worst (0.82). This behaviour is observed in the confusion matrices of Fig. 7 , indicating a larger widespread of errors compared to the previous cases. Despite that, still most of the errors are in the immediate neighbour and thus a certain tendency towards the diagonal is evidenced. Indeed, the weighted-AMAE representation demonstrate that allowing errors in the neighbourhood of the diagonal decreases AMAE to about 0.25 for $G$, to 0.20 for $B$ and to 0.58 for $R$. In this case, large errors are still encountered for $R$, but a good performance is obtained for $G$ and specially $B$, even outperforming the optimistic artificial system.

The raster plots after having employed the Gaussian regressor are depicted in the rightmost representations in Fig. 7, whereas Table 7(c) introduces the RMSE and MAE values that are obtained using the ATIC partition. Like in the ordinal regression case, the error rates provided by the Gaussian regressor are larger than in previous trials, having RMSE values ranging from 0.62 for $B$ to 0.95 for $R$. The raster plots evidence how the label 3 is not well represented, having its value centred around 2 , in all classes. For the remaining labels, this behaviour is not observed.

\subsection{Experiment 3. Clinical validation}

As explained previously, the speech therapist has been asked to perform two tasks. First, to provide blind assessments to the ATIC, SVDp and DN datasets, but redefining the GRB scale in a continuum. Second, to assess the correctness of the prediction presented in the previous experiment, and to correct those registers incorrectly labelled. After this procedure, error metrics are computed between the predicted labels 
Table 6

Experiment 2. Error measures for the ordinal classification (in a scale from 0 to 3 ).

\begin{tabular}{llll} 
Trait & Regressor & MAE & AMAE \\
\hline$G$ & POM & 0.48 & 0.50 \\
& ELMOP & 0.46 & 0.48 \\
\cline { 2 - 4 }$B$ & POM & 0.57 & 0.55 \\
& ELMOP & 0.61 & 0.59 \\
\cline { 2 - 4 }$R$ & POM & 0.64 & 0.64 \\
& ELMOP & 0.71 & 0.70 \\
\hline
\end{tabular}

(a) SVDp partition.

\begin{tabular}{llll} 
Trait & Regressor & MAE & AMAE \\
\hline$G$ & POM & 0.45 & 0.49 \\
& ELMOP & 0.40 & 0.49 \\
\cline { 2 - 4 }$B$ & POM & 0.49 & 0.48 \\
& ELMOP & 0.56 & 0.67 \\
\cline { 2 - 4 }$R$ & POM & 0.82 & 0.72 \\
& ELMOP & 0.76 & 0.71 \\
\hline
\end{tabular}

(b) DN partition.

\begin{tabular}{llll}
\hline Trait & Regressor & MAE & AMAE \\
\hline$G$ & POM & 0.65 & 0.68 \\
& ELMOP & 0.71 & 0.73 \\
\cline { 2 - 4 }$B$ & POM & 0.53 & 0.60 \\
& ELMOP & 0.58 & 0.67 \\
\hline \multirow{2}{*}{$R$} & POM & 0.64 & 0.82 \\
& ELMOP & 0.65 & 0.83 \\
\hline
\end{tabular}

(c) ATIC partition.

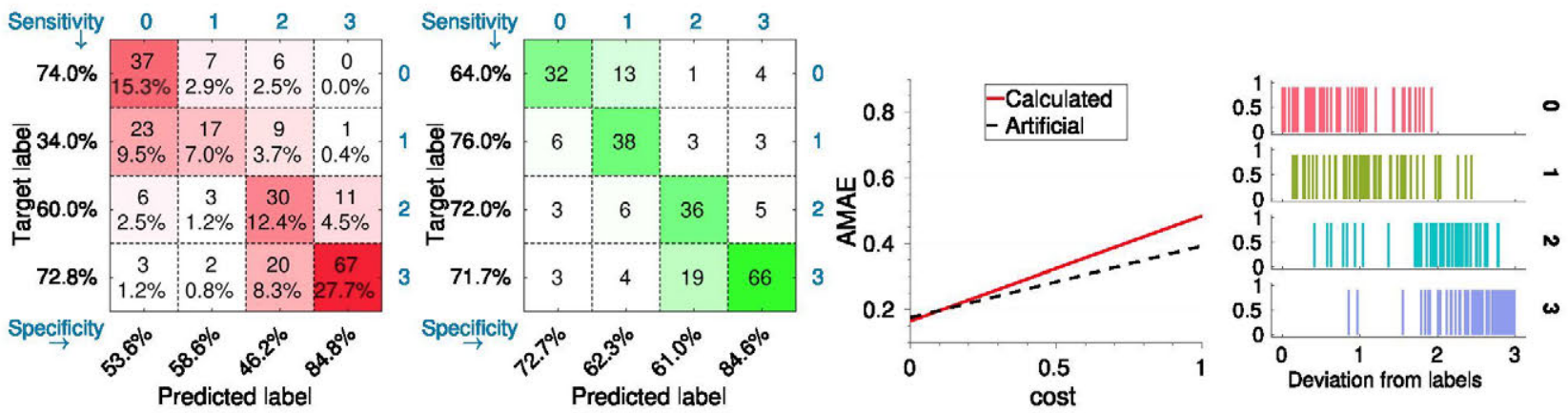

(a) Confusion matrices and weighted-AMAE for ELMOP, and raster plot of the regressor for $G$.

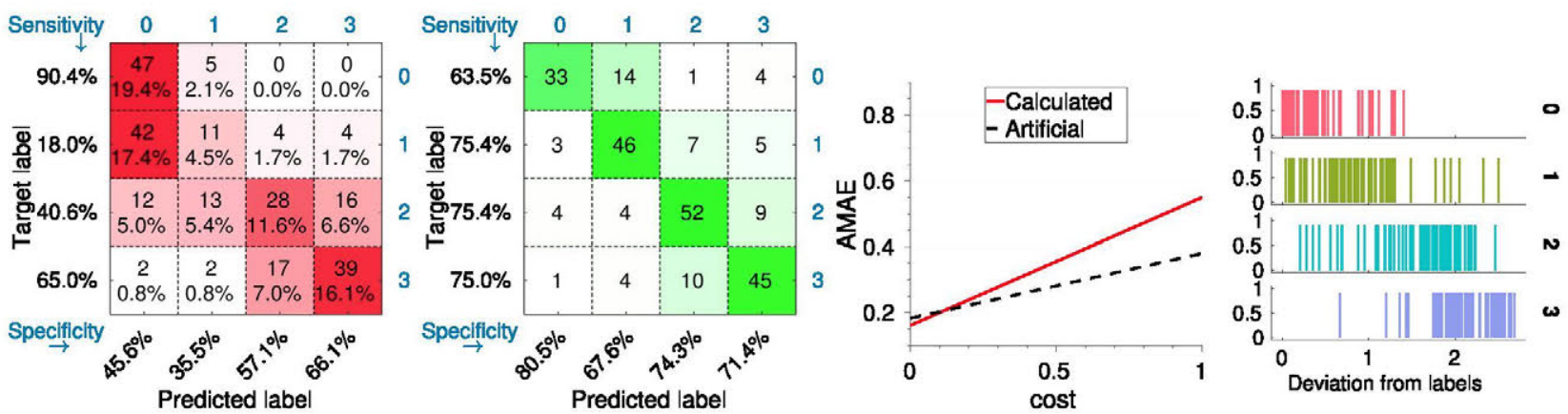

(b) Confusion matrices and weighted-AMAE for POM, and raster plot of the regressor for $B$.

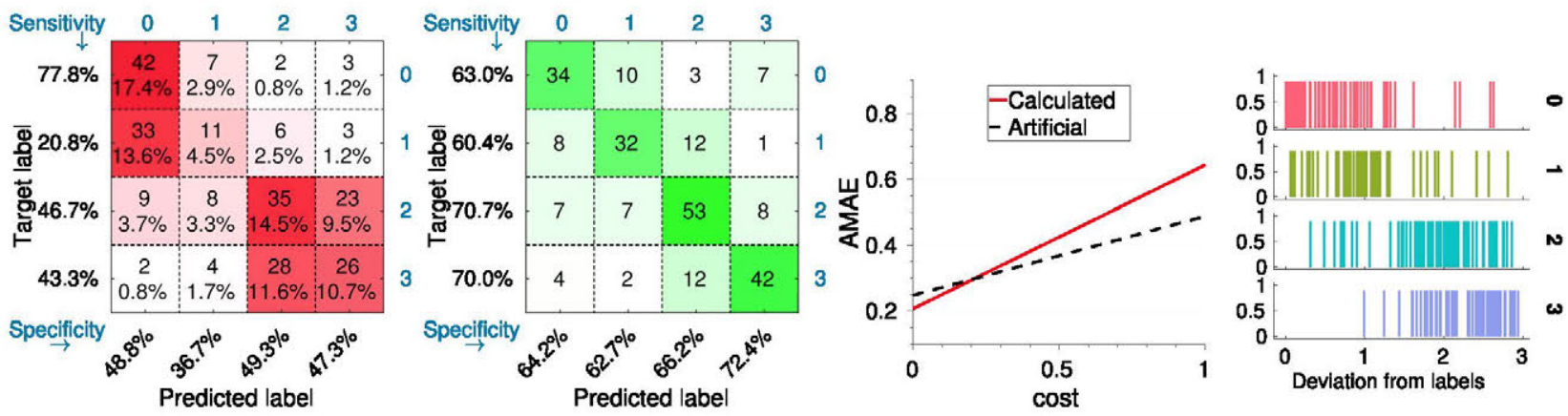

(c) Confusion matrices and weighted-AMAE for POM, and raster plot of the regressor for $R$.

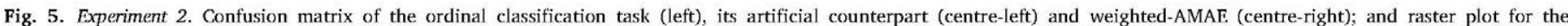

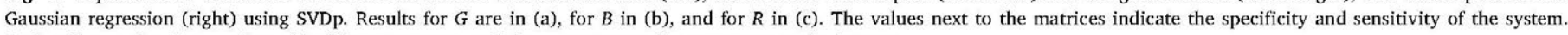
Each cell contains the number of incidences on top, and the percentage of occurrences at the bottom.

and the new evaluations corrected by the evaluator. The assessments provided by the evaluator are employed to measure its consistency.

Regarding the first task, several tests are performed in different scenarios. First, the continuous evaluations provided by the expert using the SVDp and the DN corpus are measured by means of the generalisability coefficient $(\mathcal{O})$ and the distance correlation $\left(\rho_{\mathcal{R}}\right)$. Results are presented in Table 8(a). As observed, there is a large consistency in the evaluations for all the classes and datasets, being specially large 


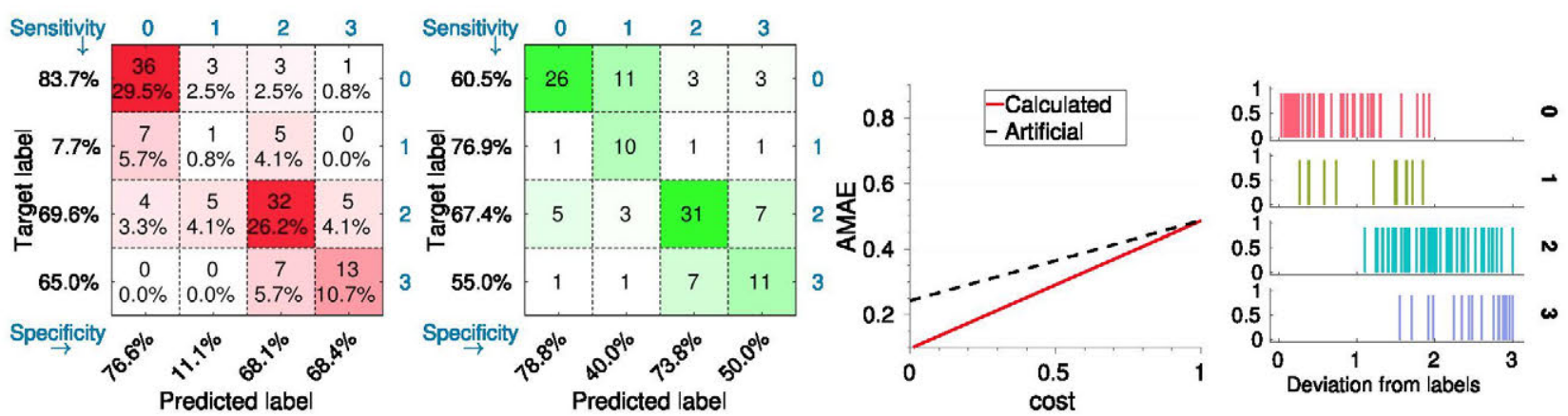

(a) Confusion matrices and weighted-AMAE for ELMOP, and raster plot of the regressor for $G$.
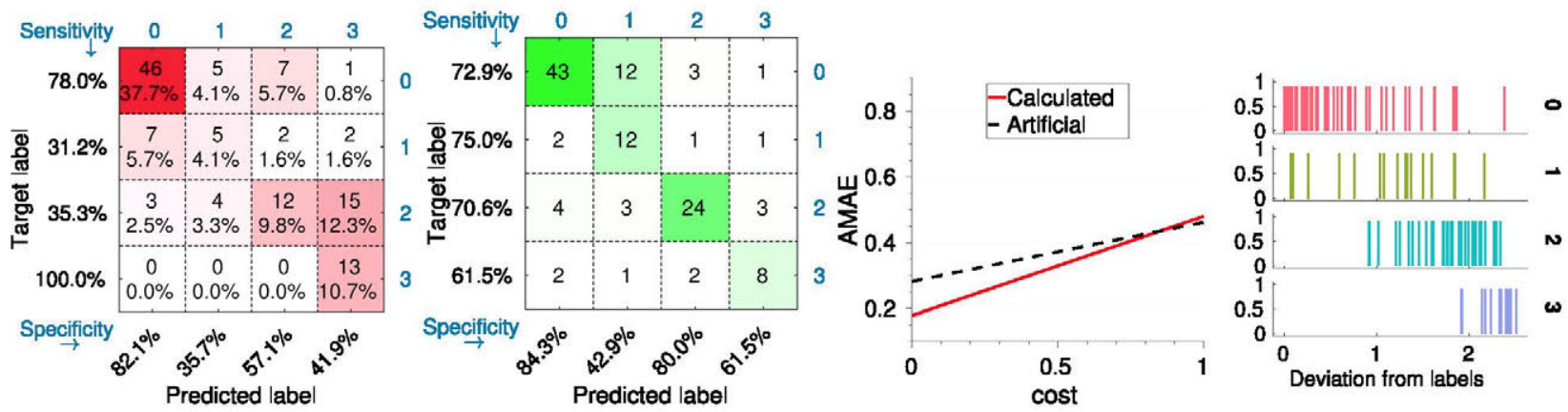

(b) Confusion matrices and weighted-AMAE for POM, and raster plot of the regressor for $B$.
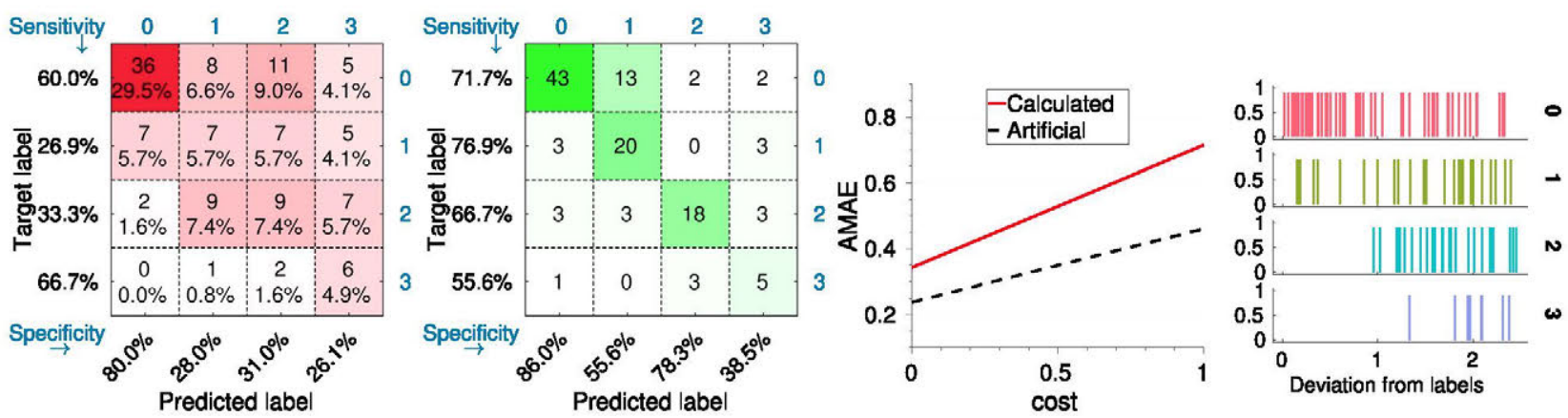

(c) Confusion matrices and weighted-AMAE for ELMOP, and raster plot of the regressor for $R$.

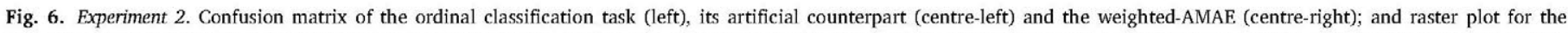

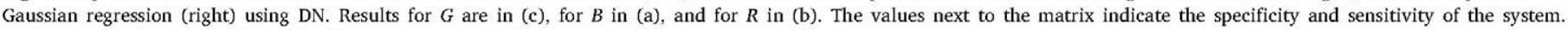
Each cell contains the number of incidences on top, and the percentage of occurrences at the bottom.

Table 7

Experiment 2. Error measures for the Gaussian regression (in a scale from 0 to 3 ).

\begin{tabular}{|c|c|c|c|c|c|c|c|c|}
\hline & RMSE & MAE & & RMSE & MAE & & RMSE & MAF \\
\hline G & 0.69 & 0.51 & $G$ & 0.69 & 0.55 & $G$ & 0.72 & 0.58 \\
\hline B & 0.72 & 0.57 & $B$ & 0.69 & 0.50 & $B$ & 0.62 & 0.45 \\
\hline$R$ & 0.76 & 0.58 & $R$ & 0.96 & 0.76 & $R$ & 0.95 & 0.70 \\
\hline \multicolumn{3}{|c|}{ (a) SVDp corpus } & \multicolumn{3}{|c|}{ (b) $\mathrm{DN}$ corpus } & \multicolumn{3}{|c|}{ (c) ATIC corpus. } \\
\hline
\end{tabular}

for $G$ where $\mho=0.94$, but with remarkably high values for $B$ ( $\mho$ up to $0.94)$ and $R(\delta$ up to 0.91$)$ too. This suggests that the speech therapist provides accurate descriptions for $G, B$ and $R$ between evaluations, even after having considered the grading process as a continuum.

To compare the consistency of these continuous gradings with the discrete labels, three corpora were used: SVDp, DN and ATIC. The results of consistency between the discrete and continuous evaluations are introduced in Table 8(b). With respect to the previous case, consistency and correlation are expected to decrease, due the comparison of the continuous values with a discrete scale, which only permits a limited number of quantisation steps (4 values). This indeed, would induce subsequently larger errors that produce a consequent decrease in consistency. Notwithstanding, for the $G$ class, the generalisability coefficient is similar when the SVD dataset is analysed. Furthermore, $\rho_{\mathcal{K}}$ maintains the same levels of correlations indicating a surprisingly large ability of the evaluator to ascertain evaluations for this particular class. When the remaining classes are analysed the expected decrease is observed. Despite that, in general terms, it can be inferred that the evaluator is consistent in her assessments.

So far these tests have provided an insight about the accuracy of the evaluator but do not provide clues about its precision. To this end, and in an attempt to correlate the results of the discrete evaluations provided by the speech therapist to those formed by a consensus of three speech pathologists, the ATIC dataset is employed. Results of this analysis are in Table 8(c). In this case, a remarkably low consistency between the evaluations of the speech therapist and the consensus of the evaluators is observed, having the largest values in $B(\mho=0.71)$. Interestingly, the worst agreement - by a large amount - is in the $R$ class $(\delta=0.52)$, which is line to what is observed in the previous two experiments. In terms of $\rho_{\mathcal{R}}$ these values vary between 0.28 to 0.48 , being indicators of poor correlation among assessments. These 


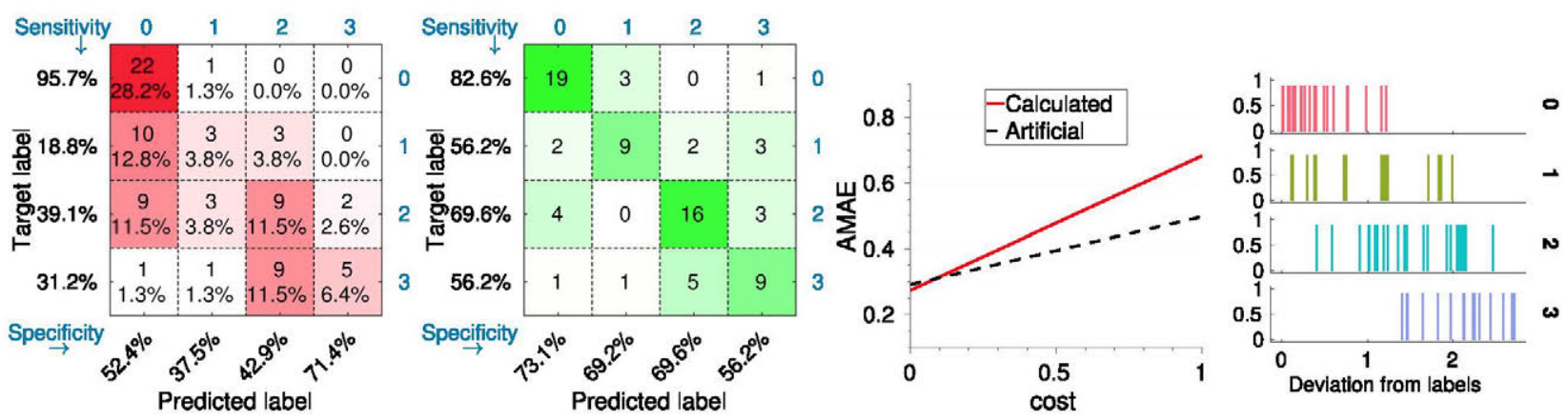

(a) Confusion matrices and weighted-AMAE for POM, and raster plot of the regressor for $G$.
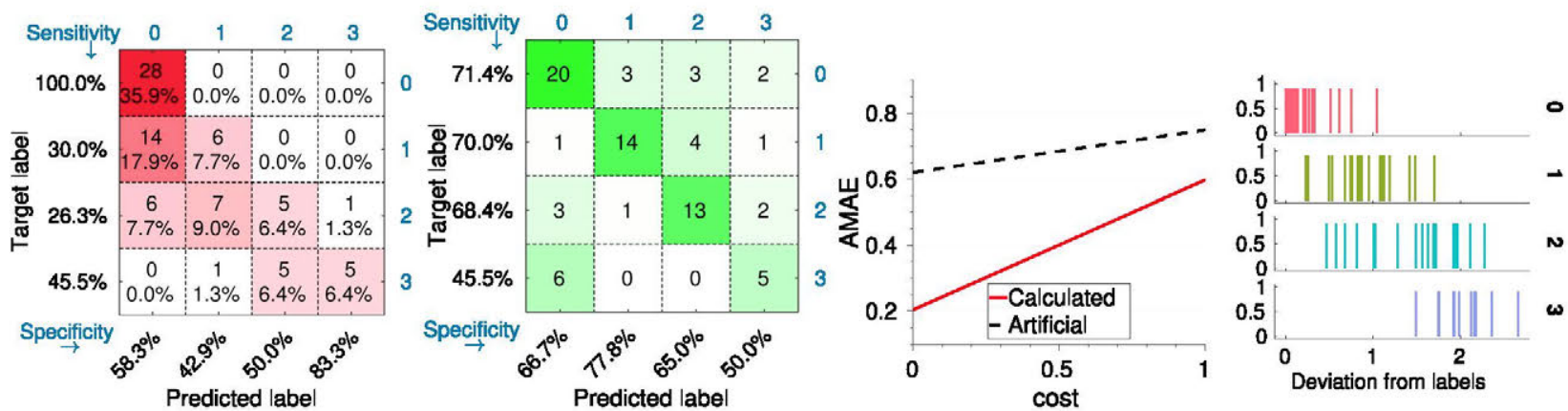

(b) Confusion matrices and weighted-AMAE for POM, and raster plot of the regressor for $B$.
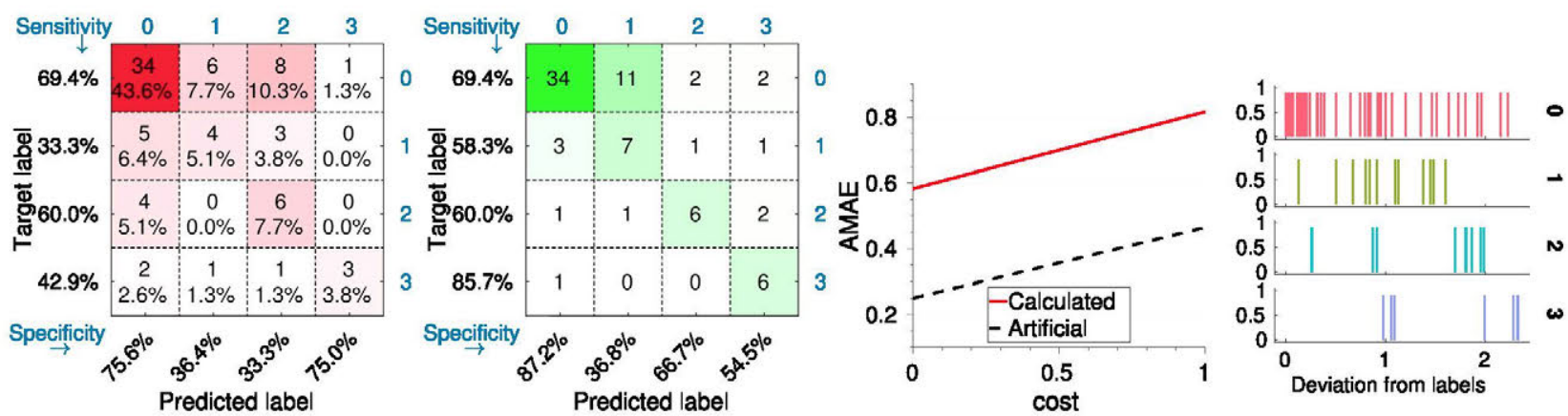

(c) Confusion matrices and weighted-AMAE for POM, and raster plot of the regressor for $R$.

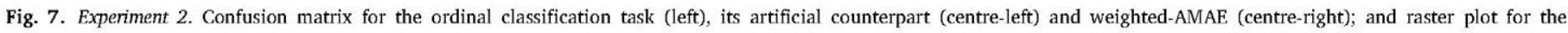

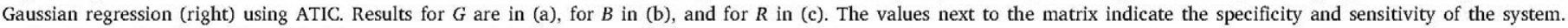
Each cell contains the number of incidences on top, and the percentage of occurrences at the bottom.

Table 8

Experiment 3. Consistency analysis of the labels.

\begin{tabular}{|c|c|c|c|c|c|c|c|c|c|c|c|c|c|c|c|c|}
\hline & \multicolumn{2}{|c|}{ SVDp } & \multicolumn{2}{|l|}{ DN } & & \multicolumn{3}{|l|}{ SVDp } & \multicolumn{3}{|l|}{ DN } & \multicolumn{2}{|l|}{ ATIC } & & \multicolumn{2}{|l|}{ ATIC } \\
\hline & \multirow[t]{2}{*}{$\bar{v}$} & \multirow[t]{2}{*}{$\rho_{\mathcal{R}}$} & \multirow[t]{2}{*}{$\bar{v}$} & \multirow[t]{2}{*}{$\rho_{\mathcal{K}}$} & & \multirow[t]{2}{*}{$\bar{v}$} & \multicolumn{2}{|l|}{$\rho_{\mathcal{R}}$} & \multirow[t]{2}{*}{$\bar{v}$} & \multicolumn{2}{|l|}{$\rho_{\text {次 }}$} & \multirow[b]{2}{*}{$\mho$} & \multirow{2}{*}{$\begin{array}{l}\rho_{\mathcal{K}} \\
\text { D. }-C_{1}\end{array}$} & & \multirow[t]{2}{*}{$\bar{v}$} & \multirow[t]{2}{*}{$\rho_{\mathcal{R}}$} \\
\hline & & & & & & & D. $-C_{1}$. & D. $-C_{2}$. & & $\overline{D .-C_{1}}$. & D. $C_{2}$. & & & & & \\
\hline$G$ & 0.93 & 0.85 & 0.94 & 0.87 & $G$ & 0.94 & 0.84 & 0.83 & 0.93 & 0.73 & 0.78 & 0.92 & 0.81 & $G$ & 0.67 & 0.48 \\
\hline$B$ & 0.89 & 0.78 & 0.94 & 0.87 & $B$ & 0.86 & 0.65 & 0.70 & 0.93 & 0.73 & 0.75 & 0.84 & 0.66 & $B$ & 0.71 & 0.62 \\
\hline$R$ & 0.91 & 0.81 & 0.85 & 0.73 & $R$ & 0.89 & 0.73 & 0.72 & 0.85 & 0.60 & 0.61 & 0.86 & 0.70 & $R$ & 0.52 & 0.28 \\
\hline \multicolumn{5}{|c|}{$\begin{array}{l}\text { (a) Consistency of the continuous } \\
\text { assessments. }\end{array}$} & \multicolumn{9}{|c|}{$\begin{array}{l}\text { (b) Consistency of discrete and continuous gradings. } D \text {. stands for discrete, } \\
C_{1} \text {. for the first continuous evaluation and } C_{2} \text {. for the second. }\end{array}$} & \multicolumn{3}{|c|}{$\begin{array}{l}\text { (c) Inter-rater } \\
\text { consistency. }\end{array}$} \\
\hline
\end{tabular}

results reveal a lack of agreement and suggest a divergence between the evaluation criteria used by the consensus of experts and by the speech therapist.

Regarding the second task presented to the evaluator, the MAE and RMSE between the labels predicted by the proposed AVCA system and the evaluations corrected by the evaluator are introduced in Table 9 for SVDp, ATIC and DN. Similarly, and to provide more hindsight about the distribution of errors, the deviation between predicted and corrected labels is presented in Fig. 8(a) for SVDp, in Fig. 8(c) for DN, and in Fig. 8(b) for ATIC.

As ascertained in Table 9, errors tend to be small in most of the cases. In particular for SVDp, even though the values of $G, B$ and $R$ are mostly satisfactory, the best performance is obtained using $G$, and the worst is observed for $R$. Likewise, RMSE varies between 0.48 (for $G$ ) to 0.67 (for $R$ ), whereas MAE is in the range of 0.32 to 0.49 . The good behaviour of $G$ is also observed in Fig. 8(a), where the raster plot is centred around 0 and a large peak is obtained in the pdf of 
Table 9

Experiment 3. Error measures between the labels predicted by the automatic system and the corrections made by the speech therapist (in a scale from 0 to 3 ).

\begin{tabular}{|c|c|c|c|c|c|c|}
\hline \multirow{2}{*}{. } & \multicolumn{2}{|l|}{$G$} & \multicolumn{2}{|l|}{$B$} & \multicolumn{2}{|l|}{$R$} \\
\hline & RMSE & MAE & RMSE & MAE & RMSE & MAE \\
\hline SVD & 0.48 & 0.32 & 0.59 & 0.47 & 0.67 & 0.49 \\
\hline DN & 0.41 & 0.29 & 0.50 & 0.38 & 0.69 & 0.48 \\
\hline ATIC & 0.46 & 0.27 & 0.47 & 0.26 & 0.50 & 0.30 \\
\hline
\end{tabular}

deviations of this class. In contrast, $R$ and $B$ present a flatter and more widespread pdf. Regarding the DN partition, RMSE varies between 0.41 for $G$, to 0.69 for $R$; whereas MAE ranges from 0.29 for $B$ to 0.48 for $R$. Similarly to SVDp, errors tend to be larger for $R$ than for the other two classes, whereas $G$ has the best general performance. This behaviour is observed in Fig. 8(c), where $G$ presents an uni-modal peak centred around zero. By contrast, $B$ and $R$ are more widespread. It is noticeable that in the raster plot of $R$, errors larger than 2 are produced. Finally for the ATIC dataset, $G, B$ and $R$ tend to behave similarly to the other two corpora. Particularly, RMSE varies between 0.46 for $G$ and 0.50 for $R$; whereas MAE ranges from 0.26 for $B$ to 0.30 for $R$. The discrepancy between the RMSE and MAE values indicates that despite errors can be considered small (labels deviate by about 0.3 units), there are cases where large errors are committed, thus increasing the RMSE values. Notwithstanding, by observing the raster plots and the pdf of deviations (Fig. 8(b)) it can be inferred that errors are centred around 0, with a large peak in this value. Similarly, it can be noticed that some large errors are committed, which in some cases are as big as 2 units (see the raster plot of the $B$ class). Despite that, the system behaves - in general terms - satisfactorily.

\section{Discussions}

This paper presents a novel methodology for modelling the perceptual capabilities of a human evaluator for assessing voices in accordance to the GRB scale. The outcomes indicate that the proposed AVCA system is capable of automatically assessing the level of dysphonia in voices according to the perceptual criteria of the human evaluator. It has been found that the most informative and consistent features amongst trials are CHNR, GNE and RALA, for all $G, B$ and $R$. Indeed, $G$ might be regarded as a class that evaluates the general state of a certain speaker i.e, it characterises the degree of hoarseness present in voice, being a good descriptor of pathology. Similarly, hoarseness has been acknowledged as a superclass that embodies roughness and breathiness perceptions simultaneously (Moers et al., 2012), and as such, is expected to embody the $B$ and $R$ perceptions too.

Ordinal classification errors in the cross-dataset experiments vary among trials, with AMAE ranging from 0.48 to 0.68 for $G$, from 0.48 to 0.60 for $B$, and from 0.64 to 0.82 for $R$. These values are of the same order of magnitude of those of MAE, for which it is possible to infer that - on average - the deviations between the predicted and the actual label are less than one label apart from each other (or half a label in the case of $G$ and $B$ ). When a Gaussian regressor is considered to account for the continuous nature of the GRB scale, errors have been found to be approximately in the same orders of magnitude of the ordinal classification. Similarly, it has been found that the largest errors are found when $R$ is analysed. This phenomenon is expected as GNE and CHNR are characteristics intended to measure additive noise that is a consequence of turbulence, i.e, they are more related to perceptual variations due to $B$ than to $R$. In a similar fashion, RALA is a parameter that measures the dispersion of energies in the MS, and as such it would be reasonable to consider it an estimator of "harmonicity", and hence, of additive noise components. Despite all three features are intended as estimators of turbulence, it is important to remark that phenomena such as jitter and shimmer, also induce a loss in periodicity that affects the harmonic components of speech. As a result, these three measures

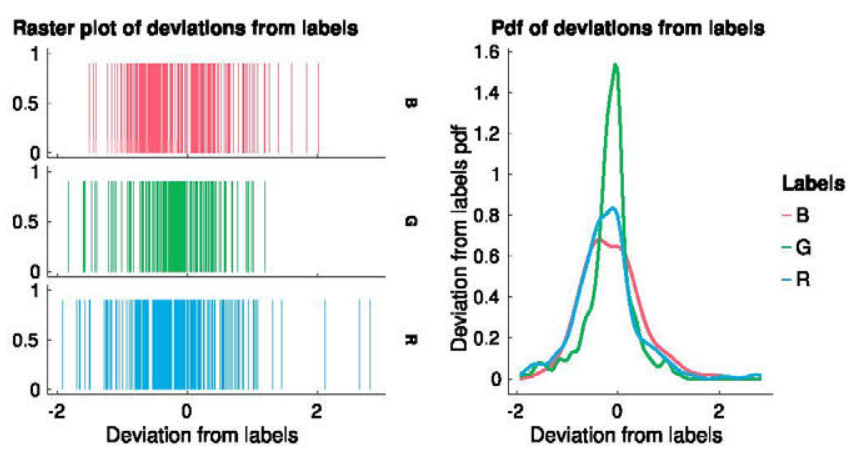

(a) SVDp dataset.
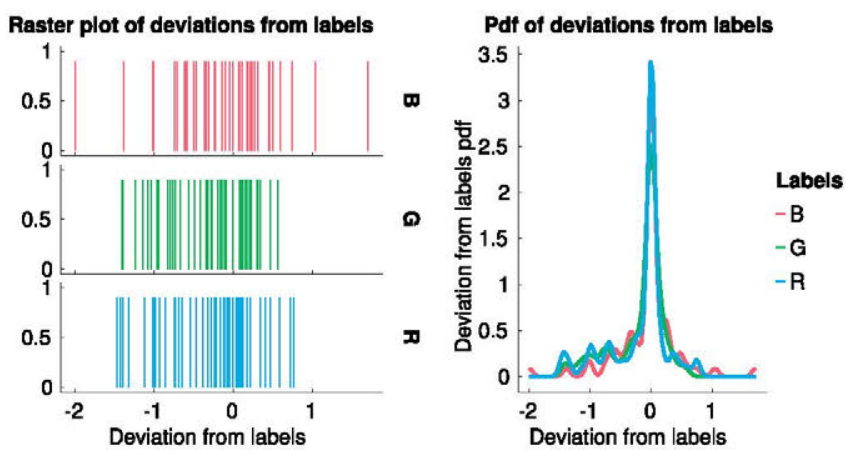

(b) ATIC dataset.

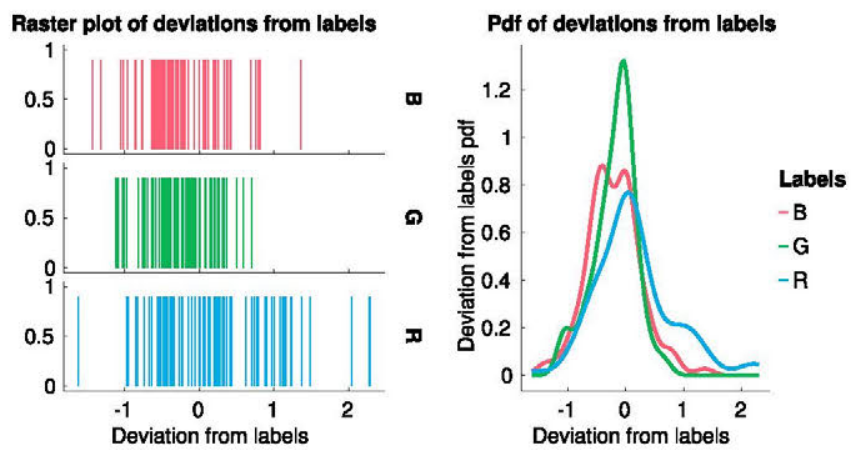

(c) DN dataset.

Fig. 8. Experiment 3. Raster plots and pdf of the deviation between the evaluations predicted by the system in experiment 2 and the labels corrected by the speech therapist for: (a) SVDp, (b) ATIC and (c) DN datasets.

are expected not only to characterise the additive noise but also to account indirectly for modulation components - as harmonic behaviour is a central part in their calculation -.

One important consideration regarding the perceptual evaluation using the GRB scale, is in the subjectivity of the evaluations. Indeed, several factors are known to affect assessments, including the evaluator's experience, the perceptual rating scale that is used, the speech task, fatigue, sensitivity of the listener to the voice that is analysed, etc. Oates (2009). Even though the corpora have been assessed by the same evaluator, the ratings are most likely affected by all these subjective factors as they are intrinsic to the evaluation process itself. In addition, it is worth noting that the evaluations of the SVD, HUPA and GMar datasets have been carried out at different times - even with some years of difference among them - for which all these above described effects might have been accentuated. Despite these shortcomings, the evaluator has shown an impressive intra-evaluation consistency, which demonstrates a well-defined evaluation criterion that is accurate and repeatable among evaluations. Nevertheless, the large differences between the assessment provided by the committee 
in ATIC and the evaluation of the speech therapist, highlights the subjectivity of the assessment problem, demonstrating the difficulties of achieving good inter-evaluator agreements. Indeed, we hypothesise that one possible reason of the decreased performance obtained with the $R$ class is attributable to the perceptual sensitivity of the evaluator to this particular class.

Outcomes also suggest that redefining the GRB scale into a continuum and having used Gaussian regression has not strongly impacted the assessment task. On one hand, the generalisability coefficient is approximately in the same order of magnitude when comparing the consistency of the continuous evaluations (Table 8(a)) to the continuous and discrete gradings (Table 8(b)). In particular, and for the first case, the worst results in terms of $\sigma$ range between 0.85 for $R$ to 0.93 for $G$, whereas for the latter it ranges between 0.84 for $R$ to 0.85 for $B$. In general these values indicate that the assessments made by the speech therapist present a large intra-evaluator agreement. On the other, the regression procedure has demonstrated reliability emulating the perceptual capabilities of the evaluator as ascertained during the clinical evaluation of results, where an agreement between the decisions taken by the expert and the ones provided by the automatic system has been demonstrated. Particularly, the largest errors between the predicted labels and the relabelled system revolves, in terms of MAE, around 0.27 for $G, 0.26$ for $B$ and 0.30 for $R$, occurring in all cases in the ATIC dataset. This is specially interesting, as the ATIC dataset presented some of the more disperse results when the Gaussian regression was considered.

Literature reports other types of systems where the automatic assessment of voice pathologies has been studied. However, a direct comparison to those studies is not possible since the methodologies followed differ drastically in terms of the techniques and of the datasets that have been used. In any case, the review of the literature suggests that all above-mentioned systems use a pattern recognition methodology that differs to the one presented in the present paper, highlighting the novelty of the approach presented in this paper, especially in terms of evaluation.

Finally, it is worth noting that the ordinal classifiers and the Gaussian regressor techniques that have been used in this paper have been chosen due to their simplicity. However, other algorithms can be tested out in order to improve performance. In particular, POM is a classical and reliable linear technique that dates back to the 80 's, but which is unable to handle nonlinearity in the feature space. Likewise, ELMOP has been found to be affected by the small dataset size (Deng et al., 2010) -as it might have been the case when the per-file statistics has been used-, and which might be the reason explaining why it has been outperformed by the simpler POM algorithm. With this in mind, other types of techniques that should be considered in the future including Gaussian processes for ordinal classification, different modifications of the SVM algorithm to handle the ordinal classification problem, or other ANN-based approaches.

\section{Conclusions}

AVCA systems have gained a great interest due to their potential applications in clinical settings, where they might turn into valuable complementary tools for improving diagnosis and screening processes. Because of the importance of the topic, the aim of this paper is to design systems capable of generalising decisions accurately about the severity of voice pathologies. To this end, a variety of trials have been performed under different settings.

It has been reported through the analysis of several sets of features and speech material of different types, that no single parameter is capable of completely characterising vocal pathology, and therefore multidimensional approaches are needed to enhance classification results. The most coherent features, as ascertained in several trials, are two estimators of perturbation noise and one descriptor of dispersion based on MS; namely, GNE, CHNR and RALA. The latter is a novel characteristic that has been recently introduced in Moro-Velázquez et al. (2015). These three parameters are consistently better than the remaining features standing as reliable predictors of $G, B, R$, and useful descriptors of dysphonic and normophonic behaviour. Even though these features are computed in different domains, they have shown complementarity. This is evidenced by the outcomes that are obtained when these three features are used jointly. In general, it has been found that the best results of the assessment system are, almost in all cases, provided by the $G$ class, whereas the worst are yielded by $R$. This phenomenon has been hypothesised to occur due to the use of GNE and CHNR, which are estimators of turbulence noise, an as such are more interrelated to perceptual variations of $B$ than to $R$.

Results also indicate that the ordinal classifier has behaved satisfactorily in all the trials, providing consistent results which are in line to those provided by the Gaussian regressor. Because of that fact, ordinal classification is recommended for the analysis of rating scales where the ordering of the assessment should be accounted. Similarly, it has been found that the distance correlation, $\rho_{\mathcal{R}}$, provides results which are consistent to other techniques used to analyse performance (such as the ordinal classifiers, feature ranking algorithms, etc.). Due to this consistency, simplicity, and its ability to characterise nonlinear relationships between variables (even in multidimensional scenarios), its usage is recommended in applications where the strength between variables should be gauged. Indeed, it can either replace or supplement other more classical correlation measures such as $\rho$, Spearman or Kendall's tau.

In most of the assessment experiments involving ordinal regression the errors occur in the immediacy of the target label. If the system is designed to accept this type of errors, a drastic drop in errors is generally obtained as ascertained by the weighted-AMAE. Outcomes also indicate that is possible to extend the discrete GRB scale to a continuum, having found evidences about the consistency that an evaluator can achieve when using this redefined scale, and about the close relationship that the continuous labels present to the standard discrete ratings. Finally, it can be concluded that it is possible to effectively emulate the perceptual capabilities of a human evaluator. Indeed, the proposed system achieves errors - between the discrete labels given by the evaluator and the predictions of the systems - which are about half an unit, on average, for the $G$ and $B$ classes, and of 0.7 for $R$. This system has also been proficient in generalising results as indicated by acceptable MAE values in the cross-dataset experiments. In addition, the clinical validation demonstrates the utility of the system to predict $G, B$ and $R$. In particular, MAE varied in the range 0.27 to 0.32 for $G, 0.26$ to 0.47 for $B$ and 0.30 to 0.49 for $R$. These values are lower than the results obtained with the proposed system, indicating that the expert considers that in most cases the predictions are in the range of what it can be considered acceptable.

As future work, other types of ordinal classifiers and regressors should be studied, such as those based on SVM, other types of ANN or Gaussian processes for ordinal regression. Moreover, it has been shown that features based on modulation spectrum constitutes an interesting source of information. For this reason we encourage an in-depth study of the modulation spectrum representations of normophonic and dysphonic conditions. The aim is to propose new characteristics that provide hindsight about vocal quality. This also includes an analysis to decompose signals into components describing pathology such as modulation, additive noise, or tremor. The methodologies proposed in this paper could be extended to the study of other types of pathologies such as those affecting speech due to neurological disorders (Parkinson's disease, spasmodic dysphonia, etc.). In particular the focus should be put into studying running speech tasks to characterise phenomena related to dysarthria, or to extract relevant information for the characterisation of phonatory and prosodic aspects of disordered speech.

\section{Acknowledgement}

This work was supported by the Ministry of Economy and Competitiveness of Spain under grant DPI2017-83405-R1. 


\section{References}

Alonso-Hernandez, J.B., De Leon, J., Alonso, I., Ferrer, M.A., 2001. Automatic detection of pathologies in the voice by HOS based parameters. EURASIP J. Appl. Signal Process. 2001 (4), 275-284. http://dx.doi.org/10.1155/S1110865701000336.

Alpan, A., Schoentgen, J., Maryn, Y., Grenez, F., Automatic perceptual categorization of disordered connected speech, in: INTERSPEECH 2010, 11th Annual Conference of the International Speech Communication Association, 2010, pp. 2574-2577.

Anniko, M., Bernal-Sprekelsen, M., Bonkowsky, V., Bradley, P., Iurato, S., 2010. Otorhinolaryngology, Head and Neck Surgery. Springer.

Arias-Londoño, J.D., Godino-Llorente, J.I., 2015. Entropies from Markov models as complexity measures of embedded attractors. Entropy 17 (6), 3595-3620. http: //dx.doi.org/10.3390/e17063595.

Arias-Londoño, J.D., Godino-Llorente, J.I., Sáenz-Lechón, N., Osma-Ruiz, V., Castellanos-Dominguez, G., 2011. Automatic detection of pathological voices using complexity measures, noise parameters, and mel-cepstral coefficients. IEEE Trans. Biomed. Eng. 58 (2), 370-379. http://dx.doi.org/10.1109/TBME.2010.2089052.

Aplicación de las tecnologías de la información y comunicaciones dataset. URL http: //www.atic.uma.es.

Atlas, L., Shamma, S.A., 2003. Joint acoustic and modulation frequency. EURASIP J. Adv. Signal Process. 2003 (7), 310290

Awan, S.N., Roy, N., Cohen, S.M., 2014. Exploring the relationship between spectral and cepstral measures of voice and the voice handicap index (VHI). J. Voice 28 (4), 430-439.

Awan, S.N., Roy, N., Dromey, C., 2009. Estimating dysphonia severity in continuous speech: application of a multi-parameter spectral/cepstral model. Clin. Linguist. Phonetics 23 (11), 825-841. http://dx.doi.org/10.3109/02699200903242988.

Baccianella, S., Esuli, A., Sebastiani, F., 2009. Evaluation measures for ordinal regression. In: 2009 Ninth International Conference on Intelligent Systems Design and Applications. IEEE, pp. 283-287. http://dx.doi.org/10.1109/ISDA.2009.230.

Bandt, C., 2005. Ordinal time series analysis. Ecol. Model. 182 (3-4), 229-238.

Barsties, B., De Bodt, M., 2015. Assessment of voice quality: Current state-of-the-art. Auris Nasus Larynx 42 (3), 183-188. http://dx.doi.org/10.1016/j.anl.2014.11.001.

Brown, G., Pocock, A., Zhao, M.-J., Luján, M., 2012. Conditional likelihood maximisation: A unifying framework for information theoretic feature selection. J. Mach. Learn. Res. 13, 27-66.

Calinon, S., 2016. A tutorial on task-parameterized movement learning and retrieval. Intel. Serv. Robot. 9 (1), 1-29. http://dx.doi.org/10.1007/s11370-015-0187-9.

Cardoso, J.S., Pinto, J.F., 2007. Learning to classify ordinal data: The data replication method. J. Mach. Learn. Res. 8, 1393-1429.

Chen, W., Wang, Z., Xie, H.-B., Yu, W., 2007. Characterization of surface EMG signal based on fuzzy entropy. IEEE Trans. Neural Syst. Rehab. Eng. 15 (2), 266-272. http://dx.doi.org/10.1109/TNSRE.2007.897025.

Cord, M., Cunningham, P. (Eds.), 2008. Machine learning techniques for multimedia. In: Cognitive Technologies. Springer Berlin Heidelberg, Berlin, Heidelberg, http: //dx.doi.org/10.1007/978-3-540-75171-7.

Cruz-Ramírez, M., Hervás-Martínez, C., Sánchez-Monedero, J., Gutiérrez, P., 2014. Metrics to guide a multi-objective evolutionary algorithm for ordinal classification. Neurocomputing 135, 21-31.

de Krom, G., 1993. A cepstrum-based technique for determining a harmonics-tonoise ratio in speech signals. J. Speech Lang. Hear. Res. 36 (2), 254-266. http: //dx.doi.org/10.1044/jshr.3602.254.

Dejonckere, P.H., 2009. Assessment of voice and respiratory function. In: Surgery of Larynx and Trachea. Springer Berlin Heidelberg, pp. 1-26.

Deng, W.-Y., Zheng, Q.-H., Lian, S.-G., Chen, L., Wang, X., 2010. Ordinal extreme learning machine. Neurocomputing 74 (1-3), 447-456.

Fredouille, C., Pouchoulin, G., Ghio, A., Revis, J., Bonastre, J.-F., Giovanni, A., 2009. Back-and-forth methodology for objective voice quality assessment: From/to expert knowledge to/from automatic classification of dysphonia. EURASIP J. Adv. Signal Process. 2009 (1), 982102.

Godino-Llorente, J.I., Osma-Ruiz, V., Sáenz-Lechón, N., Cobeta-Marco, I., GonzálezHerranz, R., Ramírez-Calvo, C., 2008. Acoustic analysis of voice using WPCVox: a comparative study with multi dimensional voice program. Eur. Arch. Oto-Rhino-Laryngol. 265 (4), 465-476.

Godino-Llorente, J.I., Sáenz-Lechón, N., Osma-Ruiz, V., Aguilera-Navarro, S., GómezVilda, P., 2006. An integrated tool for the diagnosis of voice disorders. Med. Eng. Phys. 28 (3), 276-289.

Gould, J., Waugh, J., Carding, P., Drinnan, M., 2012. A new voice rating tool for clinical practice. J. Voice 26 (4), e163-e170.

Gutierrez, P.A., Perez-Ortiz, M., Sanchez-Monedero, J., Fernandez-Navarro, F., HervasMartinez, C., 2016. Ordinal regression methods: Survey and experimental study. IEEE Trans. Knowl. Data Eng. 28 (1), 127-146.

Hermansky, H., 1990. Perceptual linear predictive (PLP) analysis of speech. J. Acoust. Soc. Am. 87, 1738.

Hillenbrand, J., Houde, R.A., 1996. Acoustic correlates of breathy vocal quality: Dysphonic voices and continuous speech. J. Speech Lang. Hear. Res. 39 (2), 311.
Kantz, H., Schreiber, T., 2004. Nonlinear Time Series Analysis, second ed. Cambridge University Press.

Kasuya, H., 1986. Normalized noise energy as an acoustic measure to evaluate pathologic voice. J. Acoust. Soc. Am. 80 (5), 1329.

Kreiman, J., Gerratt, B.R., 2010. Perceptual assessment of voice quality: Past, present, and future. Perspect. Voice Voice Disorders 20 (2), 62.

Kreiman, J., Gerratt, B.R., Kempster, G.B., Erman, A., Berke, G.S., 1993. Perceptual evaluation of voice quality. J. Speech Language Hearing Res. 36 (1), 21.

Lee, J.Y., Hahn, M., 2010. Automatic assessment of pathological voice quality using higher-order statistics in the LPC residual domain. EURASIP J. Adv. Signal Process. 2009 (1), 748207.

Little, M.A., McSharry, P.E., Roberts, S.J., Costello, D.A., Moroz, I.M., 2007. Exploiting nonlinear recurrence and fractal scaling properties for voice disorder detection. BioMed. Eng. OnLine 6 (1), 23. http://dx.doi.org/10.1186/1475-925X-6-23.

Ma, E.P.-M., Yiu, E.M.-L., 2006. Multiparametric evaluation of dysphonic severity. J. Voice 20 (3), 380-390.

Massachusetts Eye and Ear Infirmary, Voice disorders database, version.103 [cd-rom], Lincoln Park, NJ: Kay Elemetrics Corp (1994).

Michaelis, D., Gramss, T., Strube, H.W., 1997. Glottal-to-noise excitation ratio-a new measure for describing pathological voices. Acta Acust. United Acust. 83 (4), 700-706.

Moers, C., Möbius, B., Rosanowski, F., Nöth, E., Eysholdt, U., Haderlein, T., 2012. Vowel- and text-based cepstral analysis of chronic hoarseness. J. Voice 26 (4), $416-424$.

Moro-Velázquez, L., Gómez-García, J.A., Godino-Llorente, J.I., Andrade-Miranda, G., 2015. Modulation Spectra Morphological Parameters: A New Method to Assess Voice Pathologies according to the GRBAS Scale. BioMed Research International.

Oates, J., 2009. Auditory-perceptual evaluation of disordered voice quality. Folia Phoniatr. Logop. 61 (1), 49-56.

O'Brian, N., O'Brian, S., Packman, A., Onslow, M., 2003. Generalizability theory I: Assessing reliability of observational data in the communication sciences. J. Speech Lang. Hear. Res. 46 (3), 711-717. http://dx.doi.org/10.1044/1092-4388(2003/ 056).

Peng, C., Havlin, S., Stanley, H.E., Goldberger, A.L., 1995. Quantification of scaling exponents and crossover phenomena in nonstationary heartbeat time series. Chaos 5 (1), 82-87.

Pincus, S.M., 1991. Approximate entropy as a measure of system complexity. Proc. Natl. Acad. Sci. 88 (6), 2297-2301. http://dx.doi.org/10.1073/pnas.88.6.2297.

Putzer, M., Barry, W.J., 2008. Instrumental dimensioning of normal and pathological phonation using acoustic measurements. Clin. Linguist. Phonetics 22 (6), 407-420. http://dx.doi.org/10.1080/02699200701830869.

Richman, J.S., Moorman, J.R., 2000. Physiological time-series analysis using approximate entropy and sample entropy. Am. J. Physiol. Heart Circ. Physiol. 278 (6), H2039-49.

Ritchings, T., McGillion, M., Moore, C., 2002. Pathological voice quality assessment using artificial neural networks. Med. Eng. Phys. 24 (7-8), 561-564.

Rossiter, D., 2014. Statistical methods for accuracy assesment of classified thematic maps. Technical Note, Enschede, International Institute for Geo-information Science \& Earth Observation (ITC).

Saarbrüecken voice database. URL http://www.stimmdatenbank.coli.uni-saarlandde/ index.php4.

Stráník, A., Čmejla, R., Vokřál, J., 2014. Acoustic parameters for classification of breathiness in continuous speech according to the GRBAS scale. J. Voice 28 (5), 653e9-653.e17.

Stulp, F., Sigaud, O., 2015. Many regression algorithms, one unified model: A review. Neural Netw. 69, 60-79.

Sugiyama, M., 2016. Introduction to Statistical Machine Learning. Morgan Kaufmann Publishers Inc., San Francisco, CA, USA.

Székely, G.J., Rizzo, M.L., 2014. Partial distance correlation with methods for dissimilarities. Ann. Statist. 42 (6), 2382-2412.

Vaiciukynas, E., Verikas, A., Gelzinis, A., Bacauskiene, M., Minelga, J., Hållander, M., Padervinskis, E., Uloza, V., 2015. Fusing voice and query data for non-invasive detection of laryngeal disorders. Expert Syst. Appl. 42 (22), 8445-8453. http: //dx.doi.org/10.1016/j.eswa.2015.07.001.

Wang, Z., Yu, P., Yan, N., Wang, L., Ng, M.L., 2016. Automatic assessment of pathological voice quality using multidimensional acoustic analysis based on the GRBAS scale. J. Signal Process. Syst. 82 (2), 241-251. http://dx.doi.org/10.1007/ s11265-015-1016-2.

Webb, N.M., Shavelson, R.J., Haertel, E.H., 2006. Reliability Coefficients and Generalizability Theory. In: Handbook of Statistics, vol. 26, pp. 81-124.

Xie, H.-B., He, W.-X., Liu, H., 2008. Measuring time series regularity using nonlinear similarity-based sample entropy. Phys. Lett. A 372 (48), 7140-7146.

Xu, L., Wang, K., Wang, L., 2005. Gaussian kernel approximate entropy algorithm for analyzing irregularity of time-series. In: Proceedings of 2005 International Conference on Machine Learning and Cybernetics. August, pp. 5605-5608.

Zanin, M., Zunino, L., Rosso, O.A., Papo, D., 2012. Permutation entropy and its main biomedical and econophysics applications: A review. Entropy 14 (12), 1553-1577. http://dx.doi.org/10.3390/e14081553. 